\title{
Biofuel-powered soft electronic skin with multiplexed and wireless sensing for human-machine interfaces
}

\author{
You $\mathrm{Yu}^{1}$, Joanna Nassar ${ }^{1}$, Changhao Xu ${ }^{1}$, Jihong Min ${ }^{1}$, Yiran Yang ${ }^{1}$, Adam Dai ${ }^{2}$, Rohan \\ Doshi $^{2}$, Adrian Huang ${ }^{3}$, Yu Song ${ }^{1}$, Rachel Gehlhar ${ }^{4}$, Aaron D. Ames ${ }^{4}$, Wei Gao ${ }^{1,}$ \\ ${ }^{1}$ Andrew and Peggy Cherng Department of Medical Engineering, California Institute of \\ Technology, Pasadena, CA 91125, USA \\ 2Department of Electrical Engineering, California Institute of Technology, Pasadena, CA 91125, \\ USA
}

${ }^{3}$ Division of Chemistry and Chemical Engineering, California Institute of Technology, Pasadena, CA 91125, USA

${ }^{4}$ Department of Mechanical and Civil Engineering, California Institute of Technology, Pasadena, CA 91125, USA

\section{Abstract}

\begin{abstract}
Existing electronic skin (e-skin) sensing platforms are equipped to monitor physical parameters using power from batteries or near-field communication. For e-skins to be applied in the next generation of robotics and medical devices, they must operate wirelessly and be self-powered. However, despite recent efforts to harvest energy from the human body, self-powered e-skin with the ability to perform biosensing with Bluetooth communication are limited because of lack of a continuous energy source and limited power efficiency. Here, we report a flexible and fully perspiration-powered integrated electronic skin (PPES) for multiplexed metabolic sensing in situ. The battery-free e-skin contains multimodal sensors and highly efficient lactate biofuel cells that use a unique integration of zero- to three-dimensional nanomaterials to achieve high power intensity and long-term stability. The PPES delivered a record-breaking power density of 3.5 milliwatt-centimeter ${ }^{-2}$ for biofuel cells in untreated human body fluids (human sweat) and displayed a very stable performance during a 60 -hour continuous operation. It selectively monitored key metabolic analytes (e.g., urea, $\mathrm{NH}_{4}{ }^{+}$, glucose, and $\mathrm{pH}$ ) and the skin temperature during prolonged physical activities and wirelessly transmitted the data to the user interface using Bluetooth. The PPES was also able to monitor muscle contraction and work as a human-machine interface for human- prosthesis walking.
\end{abstract}

*Corresponding author. weigao@ caltech.edu.

Author contributions: W.G. and Y.Y. conceived the project. W.G. supervised the studies and Y.Y. led the experiments. J.N., C.X., and Y.Y. contributed to the system development, fabrication, and characterization; J.M., A.D., and R.D. contributed to the electronic circuit design and test. A.H. and Y.S. contributed to sensor preparation and characterization. R.G. and A.D.A. contributed to the prosthesis control study. Y. Yu, Y. Yang, and W.G. contributed the data analysis and co-wrote the paper. All authors provided feedback on the manuscript.

Competing interests: The authors declare that they have no competing financial interest.

Data and materials availability: All data needed to evaluate the conclusions in the paper are present in the paper or the Supplementary Materials. 


\section{INTRODUCTION}

Recent advances in robotics have enabled soft electronic devices at different scales with excellent biocompatibility and mechanical properties; these advances have rendered novel robotic functionalities suitable for various medical applications, such as diagnosis and drug delivery, soft surgery tools, human-machine interaction (HMI), wearable computing, health monitoring, assistive robotics, and prosthesis (1-6). Electronic skin (e-skin) can have similar characteristics to human skin, such as mechanical durability and stretchability and the ability to measure various sensations such as temperature and pressure (7-11). Moreover, e-skin can be augmented with capabilities beyond those of the normal human skin by incorporating advanced bioelectronics materials and devices. For example, the compliance of soft e-skin enables wearable sensing of biochemicals from the environment and the human body, showing great potential for wearable personalized health monitoring at molecular levels (1216).

Sophisticated tasks in practical robotic and biomedical applications-including multiplexed sensing, on-demand actuation, and wireless data transmission during prolonged operationset high standards for energy consumption (17). Batteries have been used as the primary power source of the majority of wireless e-skin systems; however, existing ones suffer from inadequate long-term continuous usability, especially when electricity is not readily available (18-20). Soft e-skins that can directly harvest energy from other accessible sources — including human motion, body heat, and solar light—are limited by their low-power density. Thus, they fail to power signal-processing circuitry and wireless data transmission (e.g., Bluetooth or radiofrequency communication) $(21,22)$. Battery-free e-skins using nearfield communication (NFC) for wireless power and data transmission with the user interface have been developed recently; however, these approaches are limited by the required proximity for data processing and readout $(23,24)$.

Human biofluids, such as human sweat, could serve as an ideal and sustainable bioenergy source for powering future e-skin devices $(25,26)$. Moreover, they contain a wealth of chemicals that can reflect an individual's health status (27-29). Recent advances in wearable sweat analysis have enabled a number of fundamental and clinical applications, such as noninvasive metabolic monitoring and disease diagnosis (30-32).

Here, we report a battery-free, fully perspiration-powered electronic skin (PPES) that harvests energy from human sweat through lactate biofuel cells (BFCs), performs continuous multiplexed monitoring of key metabolic biomarkers (e.g., glucose, urea, $\mathrm{NH}_{4}{ }^{+}$, and $\mathrm{pH}$ ), and wirelessly transmits the personalized information to a user interface via Bluetooth low energy (BLE) (Fig. 1A). Built on an ultra-soft polymeric substrate, the PPES can comply with the skin's modulus of elasticity and conformally laminate on different body parts for accurate biosensing (Fig. 1, B and C). Using cross-dimensional nanomaterial integration, the highly efficient and stable nanoengineered BFC array was able to achieve a record-breaking and sustainable high-power density, enabling continuous self-powered health monitoring. On this basis, we report the use of BFCs to fully power an e-skin with both multiplexed sensing and wireless data-transmission capabilities. The PPES was successfully validated in vivo in a cycling human trial, and its use toward noninvasive 
metabolic monitoring was further evaluated in humans involving dietary and nutrition challenges. Moreover, we demonstrate the use of the PPES as a human-machine interface for assistive robotic control: Integrated with strain sensors, the self-powered e-skin could wirelessly transmit signals to a user interface to direct a human operator to control a robotic prosthesis. This technology may substantially advance both self-powered electronic skin and personalized health care.

\section{RESULTS}

\section{Design of the soft PPES for multiplexed sensing}

The PPES consists of two main parts: (i) A nanoengineered flexible electrochemical patch contains a BFC array and a biosensor array for energy harvesting and molecular analysis in human sweat; biosensing films and biocatalytic nanomaterials are immobilized on serpentine-connected electrode arrays (Fig. 1D). (ii) A flexible electronic patch consolidates the rigid electronics on an ultrathin polyimide (PI) substrate through flexible interconnects for power management, signal processing, and wireless transmission (Fig. 1E). A skininterfaced microfluidic module was integrated into the PPES to achieve efficient fresh sweat sampling for stable BFC operation and accurate sweat analysis with high temporal resolution (Fig. 1F). The independent inlet-outlet design of the microfluidics for BFCs and sensors further minimized the potential influences of the BFC by-products on the sensing accuracy. The electronic components and interconnects of the PPES were encapsulated with polydimethyl siloxane (PDMS) to avoid sweat/electronic contact (Fig. 1F).

\section{Design and characterization of nanoengineered BFCs for highly efficient energy harvesting}

BFCs, using the enzymes as biocatalysts to transform the bioenergy into the electricity, are attractive power sources for future wearable and implantable electronics $(33,34)$. Among bioenergy resources, lactate, the main metabolic product of both muscle and brain exertion, is present in sweat at tens of millimolar levels and ideally suitable for powering future skininterfaced electronic devices $(35,36)$. However, reports of BFCs-powered wearable sensing remain very limited. The main bottlenecks/challenges for the practical use of current BFCs to power e-skin are (i) limited power density harvested from the human body (table S1) and (ii) poor BFC stability and short lifetime. Here, we used a BFC configuration that consists of lactate oxidase (LOx) immobilized bioanodes to catalyze the lactic acid to pyruvate and $\mathrm{Pt}$ alloy nanoparticle-decorated cathodes that reduce oxygen to water (Fig. 2A). Such redox reactions on BFC electrodes yield a stable current to power the electrical loads. Monolithic integration of zero-dimensional (0D) to 3D nanomaterials was used on the BFC electrodes to obtain optimal energy harvesting performance (Fig. 2, B and C).

To prepare the bioanode, we sequentially modified hierarchical Ni (h-Ni) microstructures, reduced graphene oxide (rGO) films, and Meldola's blue-tetrathiafulvalene-modified carbon nanotubes (MDB-TTF-CNT) on an Au electrode array (Fig. 2B). The extremely high current density and stability of the MDB-TTF-CNT/rGO/h-Ni bioanode illustrated in Fig. 2D and fig. S1 can be attributed to (i) the high electrochemically active surface area (ECSA) that is increased by 3000 times after nanomaterial modification (Fig. 2E, fig. S2, and 
Materials and Methods); (ii) the $\pi-\pi$ interaction between the CNTs and rGO that enhances the electron-transfer rate between LOx and electrodes; (iii) the TTF-MDB redox mediator that decreases the overpotential of the lactate oxidation reaction (text S1).

On the other hand, Pt-based nanoparticles were decorated on an MDB-modified CNT network (MDB-CNT) through electroless plating to form the BFC cathode (Fig. 2C). Note that the MDB modification was crucial to achieve uniform nanoparticle distribution with controlled sizes (figs. S3 and S4). Compared with that of the conventional bulk Pt electrode, Pt and Pt-Co nanoparticle-coated MDB-CNT electrodes showed higher ECSA of 170 and 210 times, respectively (Fig. 2F, fig. S5, and Materials and Methods) (37).

The assembled BFC array showed excellent performance, with an open-circuit potential (OCP) at $\sim 0.6 \mathrm{~V}$ and maximum power out-puts of $\sim 2.0$ and $\sim 3.5 \mathrm{~mW} \mathrm{~cm}^{-2}$ in 20 and $40 \mathrm{mM}$ lactate solutions, respectively (Fig. 2, G and $\mathrm{H}$ ). Note that the maximum power densities shifted as the lactate concentrations changed because of the varied redox reaction of MDB at different pHs (fig. S6) (38). When operating in human sweat samples, the BFCs could provide a power density as high as $3.6 \mathrm{~mW} \mathrm{~cm}^{-2}$ (Fig. 2I). This is, to the best of our knowledge, the highest power density for BFCs in untreated human body fluids (table S1).

\section{Characterization of Pt-Co nanoparticle-decorated BFC cathode for enhanced stability}

Enzymatic BFCs usually suffer from poor long-term stability, primarily due to the limited stability of their cathode. Among commonly used cathode materials, Prussian blue, $\mathrm{Ag}_{2} \mathrm{O}$, and $\mathrm{MnO}_{2}$ are consumable and cannot be recovered in situ $(39,40)$. Pt could be easily fouled during the practical uses due to the formation of the $-\mathrm{OH}$ groups on the electrode surface (Fig. 3A and fig. S7) (41); the dissociated oxidized Pt from the nanoparticles tends to redeposit on the larger particles via the Ostwald ripening mechanism (the average cohesive energy of Pt atoms in smaller nanoparticles is smaller than that in larger ones) (42). To enhance the long-term stability of the Pt particles, transition-metal dopants (i.e., Co) are introduced through electroless codeposition $(43,44)$. The resulting Pt/Co alloy nanoparticles were characterized using transmission electron microscopy (TEM) (Fig. 3B) and energydispersive $\mathrm{x}$-ray spectroscopy (fig. S8). The Pt/Co nanoparticles showed an average size of $\sim 10$ to $20 \mathrm{~nm}$ and an edge lattice spacing of $0.22 \mathrm{~nm}$ (Fig. 3B). The Co dopants could enhance the cohesive energy and thus stabilize the nanoparticles, leading to substantially reduced biofouling in body fluids and higher onset potential for oxygen reduction (45). In sweat samples, the Pt-Co/CNT showed a relatively stable onset potential compared with that of Pt/CNT (Fig. 3D). To further improve the long-term stability of the cathode in biofluids, we modified a permselective Nafion layer onto the Pt-Co/CNT (Fig. 3D). The Nafion/Pt$\mathrm{Co} / \mathrm{CNT}$ cathode showed stable performance over 2000 cycles of cyclic voltammetry (CV) scans (Fig. 3E) and a negligible fluctuation in the onset potential in a sweat sample for more than 30 hours (Fig. 3F). In addition to high stability, Pt-Co/MDB-CNT cathodes also showed the highest oxygen reduction performance compared with those modified with $\mathrm{Pt}$ or other $\mathrm{Pt}$ alloys (fig. S5), attributed to the Sabatier principle (46). 


\section{System-level integration of the PPES for energy management and signal processing}

The fully integrated PPES consists of a nanoengineered BFC array (total bioanode area, 1 $\mathrm{cm}^{2}$ ), a boost converter, a biosensor array, instrumentation amplifiers, and a programmable system on chip (PSoC) module (integrated with a BLE module, a microcontroller, and a temperature sensor) (Fig. 4A and figs. S9 and S10). The DC-DC boost converter amplifies the signal potential with a small power loss ( 20\%) (fig. S9). The output signal (3.3 V) continuously charges a capacitor $(660 \mu \mathrm{F})$ that temporarily stores the energy and powers biosensors and other electronic components. The BLE module runs in bursts of activity, periodically waking up from deep sleep mode to acquire measurements with the embedded successive-approximation analog-to-digital converter then wirelessly broadcasting the data to the user interfaces (Fig. 4B). BLE advertising is chosen here owing to the small size of the data packets and the low-power consumption.

The PPES is patterned on an ultrasoft PI substrate through a standard micro/nanofabrication process to comply with the skin's modulus of elasticity for accurate wearable biosensing (figs. S11 to S14). The fabricated via connection and serpentine-shaped interconnects in the PPES are illustrated in Fig. 4 (C to E). To minimize the overall patch size, reduce the strain, and achieve uniform strain distribution of the patch during mechanical deformation, we designed the whole BFC electrode array as a serpentine structure (fig. S15). The fully encapsulated PPES shows excellent transparency, mechanical flexibility, and stability (Fig. 4F and fig. S16).

In deep sleep mode, the whole PPES system draws a total current of $\sim 100 \mu \mathrm{A}$ at $3.3 \mathrm{~V}$, primarily from the two instrumental amplifiers (fig. S17A). The PPES is programmed to wake up periodically from deep sleep for $\sim 10 \mathrm{~ms}$ for acquiring and sending the sensing data with an average consumption of $9.35 \mathrm{~mA}$ (fig. S17B). As a result, the capacitor discharges when the PPES wakes up and is recharged within a few seconds by the BFCs. In $20 \mathrm{mM}$ lactate, dynamic changes in the potential of the capacitor on the PPES are shown in Fig. 4G. Wirelessly received BLE data in the user interface showed good agreement with the dualchannel sensor inputs (Fig. 4H). Our results demonstrate that, by using a smaller capacitor $(220$ or $400 \mu \mathrm{F}$ ), the whole PPES can be continuously powered in lactate solutions (5 to 20 $\mathrm{mM}$ ) without the need of deep sleep mode (fig. S18). Excellent long-term stability of the BFC-based electrical charging/discharging process was demonstrated by the continuous charging activity for $\sim 60$ hours, as shown in Fig. 4 (I and J) (and fig. S19): A capacitor was charged from 1.5 to $3.8 \mathrm{~V}$ continuously and repeatedly, and the charging periods could remain stable when fresh lactate fuel was supplied (fig. S19).

\section{Characterization of the PPES for multiplexed biosensing}

The PPES holds great promise for multiplexed sensing, and a particularly attractive one is wearable sweat analysis for personalized health monitoring. Important sweat biomarkerssuch as urea, glucose, $\mathrm{pH}$, and $\mathrm{NH}_{4}{ }^{+}$- contain meaningful information about an individual's physiological status. Despite high interest, only a limited number of sweat analytes can be accurately monitored by currently reported wearable sensors $(12,13,47)$. Given the complicated sweat secretion process and the electrochemical sensing mechanisms, multiplexed sensing is often essential to achieve an accurate assessment of the specific 
analyte. As an example, two sweat biosensor arrays (urea and $\mathrm{NH}_{4}{ }^{+}$sensor array and glucose and $\mathrm{pH}$ sensor array) were developed and evaluated toward metabolic monitoring.

The $\mathrm{NH}_{4}{ }^{+}$and urea sensor array was designed on the soft electrochemical patch based on the $\mathrm{NH}_{4}{ }^{+}$ion-selective electrodes (ISEs) (Fig. 5A). Compared with the $\mathrm{NH}_{4}{ }^{+}$sensor, the urea sensor contains an additional enzymatic layer where urease converts urea to carbon dioxide and ammonia; the increased $\mathrm{NH}_{4}{ }^{+}$product reflects the urea level. Figure 5 (B and $\mathrm{C}$ ) shows the potentiometric responses of the urea and $\mathrm{NH}_{4}{ }^{+}$sensors, measured in 40 to $2.5 \mathrm{mM} \mathrm{NH}_{4}{ }^{+}$ solutions and 40 to $2.5 \mathrm{mM}$ urea solutions, respectively. A linear relationship between potential output and logarithmic concentrations of the target analytes was obtained, with near-Nerstian sensitivities of 60.3 and $60.0 \mathrm{mV}$ per decade of concentration for $\mathrm{NH}_{4}{ }^{+}$and urea sensors, respectively. The sensors have good selectivity over common analytes in human sweat (fig. S20). The dependence of urea and $\mathrm{NH}_{4}{ }^{+}$concentrations on the sensor response is illustrated in Fig. 5D and fig. S21. Considering that $\mathrm{NH}_{4}{ }^{+}$level has a significant influence on urea sensor reading, it is essential to simultaneously monitor both urea and $\mathrm{NH}_{4}{ }^{+}$with real-time calibration for accurate sweat analysis.

The glucose and $\mathrm{pH}$ sensor array was prepared using a similar potentiometric sensing approach (Fig. 5E). A sandwich structure, Nafion/chitosan (CS)-glucose oxidase (GOx)/ Nafion, was coated on the platinum-deposited electrode to form a highly sensitive and selective glucose sensor; an electropolymerized polyaniline film serves as the hydrogen ionselective film for $\mathrm{pH}$ sensing. Figure 5 ( $\mathrm{F}$ and $\mathrm{G}$ ) illustrates the responses of the glucose and $\mathrm{pH}$ sensors in 40 to $200 \mu \mathrm{M}$ glucose and $\mathrm{pH} 4$ to 8 solutions, respectively. A linear response between the potential output of glucose sensor and glucose concentrations (in a physiologically relevant range of 0 to $150 \mu \mathrm{M}$ ) was obtained with a sensitivity of $0.1 \mathrm{mV} \mu \mathrm{M}$ -1 . A near-Nerstian sensitivity of $55.3 \mathrm{mV}$ per $\mathrm{pH}$ was observed for the $\mathrm{pH}$ sensor.

Considering that the glucose sensor response is heavily dependent on the solution pH (Fig. $5 \mathrm{H})$, multiplexed glucose and $\mathrm{pH}$ sensing with real-time calibration is also crucial to obtain high sensing accuracy (fig. S22).

All the sensors showed excellent long-term electrochemical and mechanical stabilities during continuous operation, indicating their promise for wearable use (figs. S23 and S24). Considering that skin temperature has a direct influence on the enzymatic biosensors (glucose and urea sensors here as shown in fig. S25), the on-chip temperature sensor in the BLE module could provide the skin temperature information for real-time calibration.

For wearable on-body use, the integration of a microfluidic module could greatly enhance the sweat sampling process and lead to a higher temporal resolution for wearable sensing and more stable power output from BFCs (Fig. 5I). The laser-patterned microfluidics was assembled in a sandwich structure (M-tape/PDMS/M-tape) and contains two reservoirs to minimize the influence of the BFC by-products on the sensing accuracy (fig. S26). An in vitro flow test showed that when the $\mathrm{NH}_{4}{ }^{+}$level in the input solution is switched from 5 to $10 \mathrm{mM}$ at a physiologically measured sweat rate of $0.05 \mathrm{ml} \mathrm{hour}^{-1}$, it takes $\sim 4 \mathrm{~min}$ for the $\mathrm{NH}_{4}{ }^{+}$sensor to reach new stable reading (Fig. 5J), indicating the small time delay for the on-body continuous monitoring. The PPES is mechanically flexible and can conformally laminate on a curved substrate (Fig. 5K). Under mechanical deformation with a bending 
curvature of $1.5 \mathrm{~cm}$ in radius, the PPES maintained consistent sensor readings (Fig. 5L). During long-period operation in human sweat samples, the PPES demonstrated stable performance in both energy harvesting (Fig. 5M) and analyte monitoring (Fig. 5N).

\section{On-body validation and evaluation of the PPES toward wearable biosensing}

On-body validation of the PPES was conducted on healthy humans toward continuous metabolic monitoring during a constant-load stationary biking exercise (Fig. 6A). Figure 6 (B and C) shows the data collected from the PPES for real-time monitoring of sweat urea, $\mathrm{NH}_{4}{ }^{+}$, glucose, and $\mathrm{pH}$. The $\mathrm{pH}$ and $\mathrm{NH}_{4}{ }^{+}$levels measured here, along with the on-chip temperature sensor readings, were also used to calibrate the readings from the glucose and urea sensors, respectively. During the biking process, the urea and $\mathrm{NH}_{4}{ }^{+}$levels in sweat decreased rapidly and then stabilized over time (Fig. 6B). A similar trend was observed for sweat glucose, whereas a stable $\mathrm{pH}$ response throughout the exercise was obtained (Fig. 6C). The PPES showed good reusability, stability, and biocompatibility during long-term usage (fig. S27).

In addition to on-body validation, the use of the PPES in metabolic and nutritional management was evaluated through controlled dietary challenges (Fig. 6, D to I). Figure 6 (D to $\mathrm{G}$ ) demonstrates that, compared with the initial levels, sweat urea and $\mathrm{NH}_{4}{ }^{+}$levels measured 2 hours after a standardized protein intake significantly increased in all three individuals. In contrast, decreased trends were obtained during the 2-hour period from all the individuals without protein intake. In an oral glucose-tolerance test, sweat glucose levels increased markedly for all individuals after the glucose intake and decreased after 2 hours for individuals without intake. These data indicate that the PPES has a great potential in selfpowered personalized physiological and metabolic monitoring.

\section{Evaluation of the PPES as a human-machine interface for robotic assistance}

HMI has attracted substantial attention over the past decade owing to its high promise in real-world biomedical applications in rehabilitation. On skin, detection of strains induced by muscle contraction using soft strain sensors integrated in e-skin systems is one promising approach to enable dynamic HMI. When integrated with soft strain sensors, the skininterfaced soft PPES could function as a human- machine interface toward robotic applications (Fig. 7A and figs. S28 and S29). The strain sensors were designed on the basis of CNTs/PDMS elastomer: The resistance of the sensors increased linearly with the applied strain (Fig. 7, B and C). Two strain sensors were placed on the hand and the elbow, respectively, and connected to the PPES (Fig. 7D); the bending of the finger and elbow could be monitored from the resistance change of the strain sensors (Fig. 7E). Each resistivetype strain sensor as part of a voltage divider consumed a total of $\sim 5 \mu \mathrm{A}$. The battery-free eskin placed on the sweaty arm was able to wirelessly control the motion of a robotic arm in real time: The robotic arm recognized the gestures of the human arm and then approached and grabbed the target object (Fig. 7F and movie S1). The PPES could also be used for robotic assistance in the rehabilitation settings. Figure $7(\mathrm{G}$ and $\mathrm{H}$ ) (and corresponding movie S2) demonstrates that prosthesis walking control could be achieved with a strain sensor-integrated PPES to achieve assistive walking in real-world environments. By incorporating more physical sensors for electroencephalogram and electromyography 
recording along with the continuous metabolic monitoring, the multimodal PPES could facilitate the design and optimization of novel prostheses that bring the human into the loop of prosthesis control to enable real-time user-specific responses to human intent and behavior.

\section{DISCUSSION}

Here, we developed a flexible, fully integrated, and self-powered e-skin platform that can provide real-time, continuous, multiplexed sensing and wirelessly transmit the data to the user interface through Bluetooth communication. Our e-skin platform provided real-time chemical sensing of $\mathrm{NH}_{4}{ }^{+}$, urea, glucose, and $\mathrm{pH}$, enhancing the capabilities of e-skin with multimodal and multianalyte detection. Moreover, the e-skin platform was able to monitor physical parameters - such as temperature, strain, and pressure-enabling self-powered wireless human-machine interaction for robotic applications such as powered prosthesis walking. The incorporation of multiplexed, multimodal chemical and physical sensing may expand the potential applications of e-skin for personalized health care.

For an e-skin to perform real-time and continuous sensing, on-board powering is necessary. Most e-skin platforms use the battery power supply, which could introduce constraints on the duration of use between charging and affect the long-lasting functionality when the electricity is not readily available. To circumvent the battery requirements, other wireless platforms have used battery-free data transmission/powering strategies, such as NFC, that require very small readout distance. The use of BLE transmission alleviates such constraints and offers a more realistic communication scheme for e-skin/wearable applications but would impose a higher power consumption. To accommodate the use of BLE, various battery-free powering strategies have been tested, such as piezo-based or biofuel-based energy harvesting. However, these strategies either have limited power densities for wearable or robotic use that fail to support the BLE functionality on a compact e-skin platform or suffer from short lifetime caused by biofouling.

The PPES proposed here has successfully resolved both challenges for on-board integration of BLE within the compact yet flexible e-skin format. With a unique integration of 0D-3D nanomaterials on bioanodes and cathodes, the BFC designed here yields a record-breaking power density (as high as $3.5 \mathrm{~mW} \mathrm{~cm}^{-2}$ in human sweat) that could support sensing and BLE functionalities on a small e-skin patch. The use of carefully designed and selected PtCo alloy nanoparticles has greatly enhanced the long-term stability of the BFC in sweat and enabled the long-term continuous use on skin.

Moreover, the developed e-skin here is a fully integrated platform with enhanced wearability and sensing accuracy. The platform is ultrathin and transparent using electronics with lowpower consumption and integrating electronics onto the soft substrate with a minimal mechanical mismatch, rendering a fully compliant e-skin for maximal comfort and wearability. The use of microfluidics significantly improves sweat refreshing and reduces interference between BFC and biosensors; a carefully studied correlation between different chemical targets yields calibrated sensor readouts that reflect higher accuracy. The systemlevel integration of electronics, microfluidics, and on-board calibration on a soft e-skin 
provides a much-enhanced accuracy of sensing results for on-body use. Moreover, the successful demonstration of the prosthetic control for robotic assistance using the PPES indicates the promise of using such platform for human machine interaction and for design/ optimization of next-generation prostheses. The development of such a multimodal, fully integrated, and self-powered platform enriches the functionalities and potentials of e-skins and opens the door to numerous robotic and wearable healthcare possibilities, such as personalized medicine.

\section{MATERIALS AND METHODS}

\section{Materials}

Graphite flake and silver nitrate were purchased from Alfa Aesar. Sodium nitrate, potassium permanganate, hydrogen peroxide, multiwalled CNTs, TTF, citric acid, chloroplatinic acid, sodium borohydride, ammonium chloride, nickel chloride, copper acetate, iron(III) chloride, copper acetate, zinc nitrate, Nafion perfluorinated resin solution (5\%), sodium thiosulfate pentahydrate, sodium bisulfite, polyvinyl chloride (PVC), polyvinyl butyral (PVB), F127, nonactin, bis(2-ethylhexyl) sebacate (DOS), 3,4-ethylenedioxythiophene (EDOT), poly(sodium 4-styrenesulfonate) (PSS), CS, PDMS, aniline, and platinum carbon black $(\mathrm{Pt} / \mathrm{C})(0.5 \%)$ were purchased from Sigma-Aldrich. Sulfuric acid, hydrochloric acid, ascorbic acid, methanol, ethanol, acetone, tetrahydrofuran, sodium chloride, disodium phosphate, urea, and dextrose (D-glucose) were purchased from Fisher Scientific. MDB was purchased from Combi- Blocks Inc. Lactate oxidase $\left(106 \mathrm{U} \mathrm{mg}^{-1}\right)$ was purchase from Toyobo Co. The CNT film and Ni substrate (h-Ni) were purchased from NTL Inc. and MXBaoheng Products, respectively. Medical tapes were purchased from Adhesives Research. PIs (PI-2611 and PI-2610) were purchased from HD MicroSystems Inc. Silver conductive paint was purchased from Structure Probe, Inc. (SPI supplies).

\section{Preparation of the BFCs}

To prepare the bioanodes, we first prepared GO suspension following the modified Hummer's method (48). Briefly, a mixture of $1 \mathrm{~g}$ of graphite flake and $23 \mathrm{ml} \mathrm{of} \mathrm{H}_{2} \mathrm{SO}_{4}$ was stirred more than 24 hours, and then $100 \mathrm{mg}$ of $\mathrm{NaNO}_{3}$ was added into the mixture. Subsequently, $3 \mathrm{~g}$ of $\mathrm{KMnO}_{4}$ was added to the mixture below $5^{\circ} \mathrm{C}$ in the ice bath. After stirring at $40^{\circ} \mathrm{C}$ for another $30 \mathrm{~min}, 46 \mathrm{ml}$ of $\mathrm{H}_{2} \mathrm{O}$ was added at $80^{\circ} \mathrm{C}$. Last, $140 \mathrm{ml}$ of $\mathrm{H}_{2} \mathrm{O}$ and $10 \mathrm{ml}$ of $\mathrm{H}_{2} \mathrm{O}_{2}(30 \%$, w/v) were introduced into the mixture to complete the reaction. The GO was washed and filtered with $1 \mathrm{M} \mathrm{HCl}$. The self-supported h-Ni was cut into 2-mmdiameter circles using a CO2 laser cutter and cleaned by ultrasonication in $4 \mathrm{M} \mathrm{HCl}$ for 30 min until the color changed from black to silver. After drying, the h-Ni substrates were immersed into a GO suspension with a concentration of $2.0 \mathrm{mg} \mathrm{ml}^{-1}$ in water for 1 hour. Then, the h-Ni substrates were transferred to $5 \mathrm{ml}$ of ascorbic acid $\left(10 \mathrm{mg} \mathrm{ml}^{-1}\right)$ overnight and heated at $75^{\circ} \mathrm{C}$ for 2 hours. After cooling down to room temperature, the rGO/h-Ni composite electrodes were rinsed with water. The free-standing CNTs were immersed into a $2 \mathrm{mM}$ MDB solution and then heated to $140^{\circ} \mathrm{C}$ overnight, followed by rinsing with water for several times. The resulted MDB- CNTs were dropcasted onto the $\mathrm{rGO} / \mathrm{h}-\mathrm{Ni}$ electrode to achieve a higher ECSA. The MDB-CNTs/rGO/h-Ni composite was soaked into the $20 \mathrm{mM}$ TTF ethanol/acetone $(9: 1, v / v)$ solution. Then, bioanodes were obtained by immersing TTF- 
MDB-CNTs/rGO/h-Ni composite into an LOx solution $\left(20 \mathrm{mg} \mathrm{ml}^{-1}\right)$ for 2 hours and dried at $4{ }^{\circ} \mathrm{C}$. Two microliters of $0.5 \%$ Nafion perfluorinated resin solution was drop-casted on the LOx/TTF-MDB-CNTs/rGO/h-Ni bioanodes to protect the enzymes during the operation.

The BFC cathodes were prepared as follows: The CNT film was first laser cut into 2-mmdiameter disks. The CNT pieces were immersed into $2 \mathrm{mM}$ MDB solution, heated to $140^{\circ} \mathrm{C}$ overnight, and then rinsed with water for several times; the MDB-CNT pieces were immersed in a $60 \mathrm{mM} \mathrm{H}_{2} \mathrm{PtCl}_{6}$ solution with $20 \mathrm{mM}$ doping metal ions (like $\mathrm{Co}, \mathrm{Ni}, \mathrm{Cu}$, and $\mathrm{Zn}$ ) and then immersed in a $0.1 \mathrm{M} \mathrm{NaBH}_{4}$ solution for seconds, followed by several times of water rinsing; $2 \mu \mathrm{l}$ of $0.5 \%$ Nafion perfluorinated resin solution was dropcasted onto the Ptor Pt alloy-decorated MDB-CNT composite surfaces.

\section{Characterizations of the BFCs}

For characterization, the scanning electron microscopy (SEM) images of the electrodes were obtained by a field-emission SEM (FEI Sirion). High-resolution TEM images were obtained by a TEM (Tecnai TF-20). CV and linear sweep voltammetry (LSV) analyses were performed through an electrochemical workstation (CHI 660E). The characterizations of BFCs were performed in McIlvaine buffer solutions ( $\mathrm{pH}$ 6.0) unless otherwise noted. $\mathrm{Ag} /$ $\mathrm{AgCl}$ references electrodes were used in the $\mathrm{BFC}$ characterization. The Pt electrode was fabricated by photolithography (Microchemicals $\mathrm{GmbH}, \mathrm{AZ}$ 9260) and electron beam (Ebeam) evaporation of $\mathrm{Cr} / \mathrm{Pt}(20 / 100 \mathrm{~nm}$, at a speed of 0.2 and $0.5 \AA / \mathrm{s}$, respectively), followed by lift-off in acetone.

The commercial Pt/C electrode was fabricated by drop-casting $5 \mu \mathrm{l} \mathrm{of} \mathrm{Pt/C} \mathrm{solution} \mathrm{(2} \mathrm{mg}$ $\mathrm{ml}^{-1}$ ) and $2.5 \mathrm{ml}$ of Nafion $(0.5 \%)$ on the Au electrode ( $3 \mathrm{~mm}$ in diameter). To quantify the ECSA of the bioanode, we tested the electrodes in McIlvaine buffer ( $\mathrm{pH}$ 6.0) with different scan rates ranging from 10 to $200 \mathrm{mV} \mathrm{s}^{-1}$. The slope of the calibration curve (the current density at $-0.1 \mathrm{~V}$ versus scan rate) was used as an estimate of the ECSA of each anode. The adsorption/desorption of hydrogen on $\mathrm{Pt}$ (here we used integral area in the $\mathrm{CV}$ in the range of -0.2 to $0.1 \mathrm{~V}$ ) was used to characterize the ECSA of the Pt-based BFC cathode. The polarization curves and power output were achieved by the LSV measurement at a scan rate of $2 \mathrm{mV} \mathrm{s}^{-1}$.

\section{Fabrication of the soft PPES}

The fabrication process of the PPES platform is illustrated in figs. S11 to S13. PI (PI-2611) was spin-coated on the silicon handling wafer with a speed of $2000 \mathrm{rpm}$ for $30 \mathrm{~s}$. Then, the PI was cured at $350^{\circ} \mathrm{C}$ for 1 hour with a ramping speed of $4{ }^{\circ} \mathrm{C} \mathrm{min}^{-1}$. The resulted PI substrate thickness was about $9 \mathrm{~mm}$. Photolithography (Microchemicals GmbH, AZ 9260) was used to define the inner connection wires. The photoresist was spin-coated on the wafer at a speed of $2400 \mathrm{rpm}$ for $30 \mathrm{~s}$ and measured to be around $10 \mathrm{~mm}$ thick. For surface treatment, reactive ion etching (RIE) [Oxford Plasmalab, 100 inductively coupled plasma (ICP)/RIE, 80 standard cubic centimeter per minute ( $\mathrm{sccm}$ ) of $\mathrm{O}_{2}, 5 \mathrm{sccm}$ of SF6, $70 \mathrm{~W}, 20$ mtorr] was used for $2 \mathrm{~min}$ to enhance surface adhesion of PI layers. E-beam evaporation of $\mathrm{Cu}\left(1.5 \mu \mathrm{m}\right.$ at a speed of $\left.2.5 \AA \mathrm{s} \mathrm{s}^{-1}\right)$ was deposited on the PI, followed by lift-off in acetone for minutes. An insulating layer of PI (PI-2610) was spin-coated on the surface with a speed 
of $5000 \mathrm{rpm}$ for $30 \mathrm{~s}$ and then cured at $350^{\circ} \mathrm{C}$ for $30 \mathrm{~min}$ with a ramping speed of $4^{\circ} \mathrm{C} \mathrm{min}$

${ }^{-1}$. The resulted intermediate PI layer thickness was about $1 \mathrm{~mm}$. Another photolithography step was used to define via connections between $\mathrm{Cu}$ layers. The wafer was selectively dryetched using ICP (Oxford Plasmalab, $100 \mathrm{ICP} / \mathrm{RIE}, 50 \mathrm{sccm}$ of $\mathrm{O}_{2}, 150 \mathrm{~W}, 80 \mathrm{mtorr}, 9 \mathrm{~min}$ ) to form via pattern. Photolithography was used to define outer connection wires. The wafer was surface-treated with RIE using the same recipe described above before metal evaporation. E-beam evaporation of $\mathrm{Cu}\left(2.5 \mu \mathrm{m}\right.$ at a speed of $\left.2.5 \AA \mathrm{s}^{-1}\right)$ was performed, followed by lift-off in acetone. Another encapsulation layer of PI (1 $\mu \mathrm{m}$ thick; PI-2610) was spin-coated, followed by fully curing. Photolithography was performed to define openings of sensors and BFC patterns, and then dry etching was performed using ICP (Oxford Plasmalab, $100 \mathrm{ICP} / \mathrm{RIE}, 50 \mathrm{sccm}$ of $\mathrm{O}_{2}, 150 \mathrm{~W}, 80 \mathrm{mtorr}, 9 \mathrm{~min}$ ).

After wiring system patterning, PI was spin-coated on the silicon handling wafer with a thickness of $9 \mu \mathrm{m}$. Photolithography (Micro-chemicals GmbH, AZ 9260) was used to define the shapes of BFCs and sensor arrays. The PI was then surface-treated with RIE to enhance surface adhesion (Oxford Plasmalab, $100 \mathrm{ICP} / \mathrm{RIE}, 80 \mathrm{sccm}$ of $\mathrm{O}_{2}, 5 \mathrm{sccm}$ of SF6, $70 \mathrm{~W}, 20$ mtorr). E-beam evaporation of $\mathrm{Cr} / \mathrm{Au}(20 / 100 \mathrm{~nm}$ at a speed of 0.2 and $0.5 \AA / s$, respectively) was performed, followed by lift-off in acetone. A thin layer of parylene (ParaTech LabTop 3000 Parylene coater) was deposited $(1 \mu \mathrm{m})$, followed by photolithography and RIE (Oxford Plasmalab, $100 \mathrm{ICP} / \mathrm{RIE}, 30 \mathrm{sccm}$ of $\mathrm{O}_{2}, 100 \mathrm{~W}, 50 \mathrm{mtorr}, 3 \mathrm{~min}$ ) to expose openings for further treatments.

\section{Assembly and characterization of the PPES}

The packaging and assembly of the PPES are illustrated in fig. S14. First, the electronic system pattern was set on $100 \mu \mathrm{m}$ of PDMS and connected with the BFC and sensor array pattern by the conductive silver paint (Structure Probe Inc.). One-hundred micrometers of PDMS was coated on the BFC and electronic system patterns. The bottom layer was cut as the BFC and sensor array pattern to expose the electrode area. After electrodes were modified on the BFC and sensor array, it was combined with the laser-cut microfluidic channels, which were assembled with two medical tape layers and a single PDMS layer in the middle.

The core of the sensor sampling and data-processing transmission system is the CYBLE-214009-00 BLE module. This microcontroller provided onboard BLE capability and 12-bit analog-to-digital converter (ADC) resolution and a minimal power consumption of $1.3 \mu \mathrm{A}$ in deep sleep. The OCP of BFCs could hardly reach $1 \mathrm{~V}$, which was significantly lower than the regular electronics needed. Thus, a soft integrated electronic patch, which contained energy boost converter for increasing the applied voltage, multiplexed sensing channels, and Bluetooth broadcast, was combined with the wearable BFCs. The Texas Instruments BQ25504 boost converter formed the core of the circuitry used to harvest energy from the BFCs. The BQ25504 was set to draw current from the BFC at 75\% of its OCP to maximize the power provided by the cell. The output of the BQ25504 was connected to a $680-\mu \mathrm{F}$ capacitor, which was set to charge to $3.3 \mathrm{~V}$ before triggering an analog switch to close, connecting the charged capacitor to the rest of the circuitry and powering the system. Then, the instrumentation amplifier and microcontroller recorded the 
response of sensor arrays and sent it to the Bluetooth module to broadcast, which were all powered under the boost voltage. The module would run in bursts of activity, periodically waking up to take ADC measurements of the sensors, convert the measurement into a voltage, and then wirelessly broadcast the data via BLE advertisement before re-entering deep sleep mode. The device was programmed through the Cypress programmable systemon-chip interface using the MiniProg3 SWD debugger and PSoC Creator 4.2 design environment. To reduce noise and eliminate the effects of impedance mismatching, instrumentation amplifiers were connected as intermediaries between the sensor electrode output and the microcontroller ADC input. The Texas Instruments INA333 was used as the instrumentation amplifier. The BLE packets broadcasted by the microcontroller were received on a computer with a CY5677 CySmart BLE 4.2 Universal Serial Bus (USB) dongle connected. The computer ran a scanner program written in C\# with the CySmart 1.3 application programming interface (API), which continually searched for an advertising device that matched the BLE address of the Cyble module, and then decoded and stored any received data.

\section{Preparation and characterization of biosensors}

To prepare the shared reference electrode of the potentiometric sensor array, we first electrodeposited $\mathrm{Ag}$ on the Au electrodes with a potentiostatic method $(-0.25 \mathrm{~V}$ for $600 \mathrm{~s})$ in the solution containing $0.25 \mathrm{M} \mathrm{AgNO}_{3}, 0.75 \mathrm{M} \mathrm{Na}_{2} \mathrm{~S}_{2} \mathrm{O}_{3}$, and $0.43 \mathrm{M} \mathrm{NaHSO}_{3}$; the $\mathrm{Ag} /$ $\mathrm{AgCl}$ electrode was obtained by dropping the $0.1 \mathrm{M} \mathrm{FeCl}_{3}$ solution on top of the $\mathrm{Ag}$ surface for $60 \mathrm{~s}$, and then the PVB reference cocktail was prepared by dissolving $79.1 \mathrm{mg}$ of PVB, $50 \mathrm{mg}$ of $\mathrm{NaCl}, 1 \mathrm{mg}$ of F127, and $0.2 \mathrm{mg}$ of multiwalled CNT in $1 \mathrm{ml}$ of methanol; a $6.6 \mu \mathrm{l}$ of reference cocktail was modified on the $\mathrm{Ag} / \mathrm{AgCl}$ electrode and left overnight.

The $\mathrm{NH}_{4}{ }^{+}$selective electrode was prepared as follows: $100 \mathrm{mg}$ of the $\mathrm{NH}_{4}{ }^{+}$selective membrane cocktail consisting of $1 \% \mathrm{NH}_{4}{ }^{+}$ionophore (nonactin), 33\% PVC, and 66\% DOS (w/w) was dissolved in $660 \mu \mathrm{l}$ of tetrahydrofuran. The membrane cocktail was stored at $4^{\circ} \mathrm{C}$. A constant current of $0.2 \mathrm{~mA} \mathrm{~cm}^{-2}$ was applied to electrodeposit the poly(EDOT) polystyrene sulfonate (PEDOT:PSS) membrane on the Au electrode in the solution containing 0.01 M EDOT and 0.1 M NaPSS to minimize the potential drift of the ISEs. Cocktail solution $(6.6 \mu \mathrm{l})$ was dropcasted over the PEDOT layer to create an $\mathrm{NH}_{4}{ }^{+}$selective membrane. The modified electrodes were left drying overnight. To prepare urea-sensing electrode, we dropcasted $3.7 \mu \mathrm{l}$ of urease solution $\left(10 \mathrm{mg} \mathrm{ml}^{-1}\right)$ onto the $\mathrm{NH}_{4}{ }^{+}$ISE four times, and then $3.3 \mu \mathrm{l}$ of $0.5 \%$ Nafion perfluorinated resin solution was dropped over the sensor area. The modified sensors were dried at $4^{\circ} \mathrm{C}$ overnight.

To prepare the glucose sensor, we used differential pulse amperometry (100 cycles in total) to electrodeposit the Pt on the Au electrode. A $-0.4-\mathrm{V}$ potential was applied for $1 \mathrm{~s}$, and 1.0 $\mathrm{V}$ was used as the cleaning voltage for $0.5 \mathrm{~s}$. A $1 \%$ Nafion (1.1 $\mu \mathrm{l}$; prepared by dilution of Nafion perfluorinated resin solution in water) was dropped on the Pt surface, and $2 \mu \mathrm{l}$ of CSGOx mixture (3:1, v/v) was modified on the electrode. The potentiometric glucose sensors were dried at $4{ }^{\circ} \mathrm{C}$ overnight, and then another $1.1 \mu \mathrm{l}$ of $1 \%$ Nafion (1\%) was dropped, covering the enzymes to form the sandwich structure. For the $\mathrm{pH}$ sensor, the polyaniline was 
electropolymerized on the Au electrodes in a solution containing $0.1 \mathrm{M}$ aniline and $0.1 \mathrm{M}$ $\mathrm{HCl}$ using $\mathrm{CV}$ from -0.2 to $1 \mathrm{~V}$ for 50 cycles at a scan rate of $50 \mathrm{mV} \mathrm{s}^{-1}$.

For the in vitro sensor characterizations, analyte solutions were prepared in Mcllvaine buffer solutions ( $\mathrm{pH} 6.0$ for urea, glucose, and $\mathrm{NH}_{4}{ }^{+}$). To obtain the best performance for longterm continuous measurements, we placed the $\mathrm{NH}_{4}{ }^{+}$ion-selective sensors and urea sensors in a solution containing $0.1 \mathrm{M} \mathrm{NH} 4 \mathrm{Cl}$ and $20 \mathrm{mM}$ urea for 1 hour before measurements. The glucose and $\mathrm{pH}$ sensors were placed in a solution containing $100 \mu \mathrm{M}$ glucose and $\mathrm{H}_{2} \mathrm{O}$ for 1 hours, separately. This conditioning process greatly helps to minimize the potential drift.

\section{Preparation of the strain sensors}

CNT-PDMS elastomer was produced by the solution-evaporation method. CNTs $(7 \% \mathrm{w} / \mathrm{w})$ were added to SYLGARD 184 silicone elastomer base and toluene mixture $(1: 4 \mathrm{v} / \mathrm{v})$ at room temperature. Then, the mixture was poured into a culture dish. After toluene was evaporated, uncured CNT-PDMS was mixed with curing agent (10:1) and poured onto the mask made by tape and scraped flat with a glass slide. After the mask was removed, the CNT-PDMS was baked at $80^{\circ} \mathrm{C}$ for 1 hour. Then, uncured EcoFlex was spin-coated on it and cured at $80^{\circ} \mathrm{C}$ for 1 hour. The silver paste was used to link the pad on the patterned CNT-PDMS with thin wires. Another layer of EcoFlex was spin-coated for encapsulation and protection. The strain sensors were then connected to the PPES through external wires.

\section{On-body evaluation of the PPES for wearable sensing}

The validation and evaluation of the lab-on-skin platform were performed using humans in compliance with a protocol (ID: 19-0892) that was approved by the Institutional Review Board (IRB) at California Institute of Technology (Caltech). The participating individuals (age range, 18 to 65) were recruited from the Caltech campus and the neighboring communities through advertisement by posted notices, word of mouth, and email distribution. All individuals gave written informed consent before participation in the study.

To validate the PPES, constant-load cycling exercise was conducted on three individuals. A stationary exercise bike (Kettler Axos Cycle M-LA) was used for cycling trials. The individuals reported to the laboratory with overnight fasting. The individuals' foreheads were cleaned with water and alcohol swabs before the PPES was placed. The individuals cycled at $60 \mathrm{rpm}$ for $40 \mathrm{~min}$, followed by a 10-min cooldown session. When the sweat filled the BFC reservoir, the PPES started to transmit the sensing data to the user interface through the Bluetooth every $15 \mathrm{~s}$, and the data were further converted to the concentration levels after temperature and sweat analyte calibrations. During biking, the sweat would continuously refill both the BFC and the sensor array reservoir. For each diary challenge study, after the first cycling trial, the individuals were given a standardized protein drink ( $30 \mathrm{~g}$ of protein) for urea and $\mathrm{NH}_{4}{ }^{+}$test and three energy bars (66 $\mathrm{g}$ of total sugars) for glucose, respectively. After 2 hours, the individuals cycled at $60 \mathrm{rpm}$ for $40 \mathrm{~min}$, followed by a 10-min cooldown session, and the PPES would monitor the sweat component change. The first set of data received by the PPES was plotted in Fig. 6 (D to I). 


\section{Evaluation of the PPES as a human-machine interface for robotic assistance}

For robotic arm control, two strain sensors were set on the hand and the elbow of a healthy individual with medical tape, respectively. The individual's arm was cleaned with water and alcohol swabs before the PPES was placed. The individual cycled at $60 \mathrm{rpm}$ for $10 \mathrm{~min}$. When the sweat filled the BFC reservoir, the PPES wirelessly transmitted the strain sensor data to the user interface, and the data were further used to control the robotic arm.

For the PPES-based prosthesis control study, a strain sensor was set on the elbow of a healthy individual with medical tape and connected with the PPES. When filled with $20 \mathrm{mM}$ lactate, the PPES wirelessly transmitted the measured strain sensor data to the user interface. When the sensor reached a certain threshold, a human operator sent a computer command through USB to the custom- built powered transfemoral prosthesis, AMPRO3 (49), to trigger the start of one swing step. With a proportional derivative (PD) controller, the prosthesis tracked a time-based trajectory designed with the methods in (50). This research was approved by Caltech IRB with a protocol (ID: 16-0693) for human subject testing.

\section{Supplementary Material}

Refer to Web version on PubMed Central for supplementary material.

\section{Funding}

This work was supported by California Institute of Technology Startup Grant, the Rothenberg Innovation Initiative $\left(\mathrm{RI}^{2}\right)$ program, the Carver Mead New Adventures Fund, and NIH (no. 5R21NR018271) (all to W.G.). We gratefully acknowledge critical support and infrastructure provided for this work by the Kavli Nanoscience Institute at Caltech.

\section{REFERENCES AND NOTES}

1. Yang G-Z, Bellingham J, Dupont PE, Fischer P, Floridi L, Full R, Jacobstein N, Kumar V, McNutt M, Merrifield R, Nelson BJ, Scassellati B, Taddeo M, Taylor R, Veloso M, Wang ZL, Wood R, The grand challenges of Science Robotics. Sci. Robot 3, eaar7650 (2018).

2. Someya T, Amagai M, Toward a new generation of smart skins. Nat. Biotechnol 37, 382-388 (2019). [PubMed: 30940942]

3. Xu S, Zhang Y, Jia L, Mathewson KE, Jang K-I, Kim J, Fu H, Huang X, Chava P, Wang R, Bhole S, Wang L, Na YJ, Guan Y, Flavin M, Han Z, Huang Y, Rogers JA, Soft microfluidic assemblies of sensors, circuits, and radios for the skin. Science 344, 70-74 (2014). [PubMed: 24700852]

4. Rich SI, Wood RJ, Majidi C, Untethered soft robotics. Nat. Electron 1, 102-112 (2018).

5. Hammock ML, Chortos A, Tee BC-K, Tok JB-H, Bao Z, 25th anniversary article: The evolution of electronic skin (e-skin): A brief history, design considerations, and recent progress. Adv. Mater 25, 5997-6038 (2013). [PubMed: 24151185]

6. Laschi C, Mazzolai B, Cianchetti M, Soft robotics: Technologies and systems pushing the boundaries of robot abilities. Sci. Robot 1, eaah3690 (2016).

7. Chortos A, Liu J, Bao Z, Pursuing prosthetic electronic skin. Nat. Mater 15, 937-950 (2016). [PubMed: 27376685]

8. Gong S, Schwalb W, Wang Y, Chen Y, Tang Y, Si J, Shirinzadeh B, Cheng W, A wearable and highly sensitive pressure sensor with ultrathin gold nanowires. Nat. Commun 5, 3132-3140 (2014). [PubMed: 24495897]

9. Wang X, Dong L, Zhang H, Yu R, Pan C, Wang ZL, Recent progress in electronic skin. Adv. Sci 2, 1500169-1500190 (2015). 
10. Liu Y, Liu J, Chen S, Lei T, Kim Y, Niu S, Wang H, Wang X, Foudeh AM, Tok JB, Bao Z, Soft and elastic hydrogel-based microelectronics for localized low-voltage neuromodulation. Nat. Biomed. Eng 3, 58-68 (2019). [PubMed: 30932073]

11. Son D, Lee J, Qiao S, Ghaffari R, Kim J, Lee JE, Song C, Kim SJ, Lee DJ, Jun SW, Yang S, Park M, Shin J, Do K, Lee M, Kang K, Hwang CS, Lu N, Hyeon T, Kim DH, Multifunctional wearable devices for diagnosis and therapy of movement disorders. Nat. Nanotechnol 9, 397-404 (2014). [PubMed: 24681776]

12. Gao W, Emaminejad S, Nyein HYY, Challa S, Chen K, Peck A, Fahad HM, Ota H, Shiraki D. Kiriya, Lien D-H, Brooks GA, Davis RW, Javey A, Fully integrated wearable sensor arrays for multiplexed in situ perspiration analysis. Nature 529, 509-514 (2016). [PubMed: 26819044]

13. Bariya M, Nyein HYY, Javey A, Wearable sweat sensors. Nat. Electron 1, 160-171 (2018).

14. Kim J, Campbell AS, de Avila BE-F, Wang J, Wearable biosensors for healthcare monitoring. Nat. Biotechnol 37, 389-406 (2019). [PubMed: 30804534]

15. Yang Y, Gao W, Wearable and flexible electronics for continuous molecular monitoring. Chem. Soc. Rev 48, 1465-1491 (2019). [PubMed: 29611861]

16. Nakata S, Shiomi M, Fujita Y, Arie T, Akita S, Takei K, A wearable pH sensor with high sensitivity based on a flexible charge-coupled device. Nat. Electron 1, 596-603 (2018).

17. Park S, Heo SW, Lee W, Inoue D, Jiang Z, Yu K, Jinno H, Hashizume D, Sekinoi M, Yokota T, Fukuda K, Tajima K, Someya T, Self-powered ultra-flexible electronics via nano-grating-patterned organic photovoltaics. Nature 561,516-521 (2018). [PubMed: 30258137]

18. Zeng W, Shu L, Li Q, Chen S, Wang F, Tao X-M, Fiber-based wearable electronics: A review of materials, fabrication, devices, and applications. Adv. Mater 26, 5310-5336 (2014). [PubMed: 24943999]

19. Zamarayeva AM, Ostfeld AE, Wang M, Duey JK, Deckman I, Lechêne BP, Davies G, Steingart DA, Arias AC, Flexible and stretchable power sources for wearable electronics. Sci. Adv 3, e1602051 (2017). [PubMed: 28630897]

20. Li J, Zhao J, Rogers JA, Materials and designs for power supply systems in skin-interfaced electronics. Acc. Chem. Res 52, 53-62 (2019). [PubMed: 30525449]

21. Chen J, Huang Y, Zhang N, Zou H, Liu R, Tao C, Fan X, Wang ZL, Micro-cable structured textile for simultaneously harvesting solar and mechanical energy. Nat. Energy 1, 16138-16146 (2016).

22. Suarez F, Nozariasbmarz A, Vashaee D, Öztürk MC, Designing thermoelectric generators for selfpowered wearable electronics. Energ. Environ. Sci 9, 2099-2113 (2016).

23. Lou Z, Li L, Wang L, Shen G, Recent progress of self-powered sensing systems for wearable electronics. Small 13, 1701791-1701818 (2017).

24. Bandodkar AJ, Gutruf P, Choi J, Lee K, Sekine Y, Reeder JT, Jeang WJ, Aranyosi AJ, Lee SP, Model JB, Ghaffari R, Su C-J, Leshock JP, Ray T, Verrillo A, Thomas K, Krishnamurthi V, Han S, Kim J, Krishnan S, Hang T, Rogers JA, Battery-free, skin-interfaced microfluidic/electronic systems for simultaneous electrochemical, colorimetric, and volumetric analysis of sweat. Sci. Adv 5, eaav3294 (2019). [PubMed: 30746477]

25. Escalona-Villalpando RA, Ortiz-Ortega E, Bocanegra-Ugalde JP, Minteer SD, Ledesma-García J, Arriaga LG, Clean energy from human sweat using an enzymatic patch. J. Power Sources 412, 496-504 (2019).

26. Bandodkar AJ, You J-M, Kim N-H, Gu Y, Kumar R, Mohan AMV, Kurniawan J, Imani S, Nakagawa T, Parish B, Parthasarathy M, Mercier PP, Xu S, Wang J, Soft, stretchable, high power density electronic skin-based biofuel cells for scavenging energy from human sweat. Energ. Environ. Sci 10, 1581-1589 (2017).

27. Guo H, Pu X, Chen J, Meng Y, Yeh M-H, Liu G, Tang Q, Chen B, Liu D, Qi S, Wu C, Hu C, Wang J, Wang ZL, A highly sensitive, self-powered triboelectric auditory sensor for social robotics and hearing aids. Sci. Robot 3, eaat2516 (2018).

28. Heikenfeld J, Jajack A, Feldman B, Granger SW, Gaitonde S, Begtrup G, Katchman BA, Accessing analytes in biofluids for peripheral biochemical monitoring. Nat. Biotechnol 37, 407419 (2019). [PubMed: 30804536]

29. Suhre K, Shin S-Y, Petersen A-K, Mohney RP, Meredith D, Wagele B, Altmaier E; CARDIoGRAM, Deloukas P, Erdmann J, Grundberg E, Hammond CJ, de Angelis MH, 
Kastenmuller G, Kottgen A, Kronenberg F, Mangino M, Meisinger C, Meitinger T, Mewes H-W, Milburn MV, Prehn C, Raffler J, Ried JS, Romisch-Margl W, Samani NJ, Small KS, Wichmann HE, Zhai G, Illig T, Spector TD, Adamski J, Soranzo N, Gieger C, Human metabolic individuality in biomedical and pharmaceutical research. Nature 477, 54-60 (2011). [PubMed: 21886157]

30. Koh A, Kang D, Xue Y, Lee S, Pielak RM, Kim J, Hwang T, Min S, Banks A, Bastien P, Manco MC, Wang L, Ammann KR, Jang K-I, Won P, Han S, Ghaffari R, Paik U, Slepian MJ, Balooch G, Huang Y, Rogers JA, A soft, wearable microfluidic device for the capture, storage, and colorimetric sensing of sweat. Sci. Transl. Med 8, 366ra165 (2016).

31. Meng B, Tang W, Too Z.-h., Zhang X, Han M, Liu W, Zhang H, A transparent single-frictionsurface triboelectric generator and self-powered touch sensor. Energ. Environ. Sci 6, 3235-3240 (2013).

32. Lee H, Choi TK, Lee YB, Cho HR, Ghaffari R, Wang L, Choi HJ, Chung TD, Lu N, Hyeon T, Choi SH, Kim DH, A graphene-based electrochemical device with thermoresponsive microneedles for diabetes monitoring and therapy. Nat. Nanotechnol 11, 566-572 (2016). [PubMed: 26999482]

33. Xiao X, Xia H.-q., Wu R, Bai L, Yan L, Magner E, Cosnier S, Lojou E, Zhu Z, Liu A, Tackling the challenges of enzymatic (bio)fuel cells. Chem. Rev 119, 9509-9558 (2019). [PubMed: 31243999]

34. Jia W, Valdes-Ramirez G, Bandodkar AJ, Windmiller JR, Wang J, Epidermal biofuel cells: Energy harvesting from human perspiration. Angew. Chem. Int. Ed 52, 7233-7236 (2013).

35. Dienel GA, Hertz L, Glucose and lactate metabolism during brain activation. J. Neurosci. Res 66, 824-838 (2001). [PubMed: 11746408]

36. Barros LF, Metabolic signaling by lactate in the brain. Trends Neurosci. 36, 396-404 (2013). [PubMed: 23639382]

37. McCrum IT, Janik MJ, Deconvoluting cyclic voltammograms to accurately calculate Pt electrochemically active surface area. J. Phys. Chem. C 121,6237-6245 (2017).

38. Ferreira CU, Gushikem Y, Kubota LT, Electrochemical properties of Meldola's Blue immobilized on silica-titania phosphate prepared by the sol-gel method. J. Solid State Electrochem 4, 298-303 (2000).

39. Xie X, Ye M, Hsu P-C, Liu N, Criddle CS, Cui Y, Microbial battery for efficient energy recovery. Proc. Natl. Acad. Sci. U.S.A 110, 15925-15930 (2013). [PubMed: 24043800]

40. Bai L, Jin L, Han L, Dong S, Self-powered fluorescence controlled switch systems based on biofuel cells. Energ. Environ. Sci 6, 3015-3021 (2013).

41. Vogel W, Lundquist L, Ross P, Stonehart P, Reaction pathways and poisons-II. Electrochim. Acta 20, 79-93 (1975).

42. Stephens IEL, Bondarenko AS, Grønbjerg U, Rossmeisl J, Chorkendorff I, Understanding the electrocatalysis of oxygen reduction on platinum and its alloys. Energ. Environ. Sci 5, 6744-6762 (2012).

43. Nie Y, Li L, Wei Z, Recent advancements in Pt and Pt-free catalysts for oxygen reduction reaction. Chem. Soc. Rev 44, 2168-2201 (2015). [PubMed: 25652755]

44. Greeley J, Stephens IEL, Bondarenko AS, Johansson TP, Hansen HA, Jaramillo TF, Rossmeisl J, Chorkendorff I, Nørskov JK, Alloys of platinum and early transition metals as oxygen reduction electrocatalysts. Nat. Chem 1, 552-556 (2009). [PubMed: 21378936]

45. Zhang B-W, Yang H-L, Wang Y-X, Dou S-X, Liu H-K, A comprehensive review on controlling surface composition of Pt-based bimetallic electrocatalysts. Adv. Energy Mater 8, 17035971703614 (2018).

46. Stamenkovic VR, Mun BS, Arenz M, Mayrhofer KJJ, Lucas CA, Wang G, Ross PN, Markovic NM, Trends in electrocatalysis on extended and nanoscale Pt-bimetallic alloy surfaces. Nat. Mater 6, 241-247 (2007). [PubMed: 17310139]

47. Yang Y, Song Y, Bo X, Min J, Pak OS, Zhu L, Wang M, Tu J, Kogan A, Zhang H, Hsiai TK, Li Z, Gao W, A laser-engraved wearable sensor for sensitive detection of uric acid and tyrosine in sweat. Nat. Biotechnol 38, 217-224 (2020). [PubMed: 31768044]

48. Hummers WS Jr., Offeman RE, Preparation of graphitic oxide. J. Am. Chem. Soc 80, 1339-1339 (1958).

49. Zhao H, Horn J, Reher J, Paredes V, Ames AD, First steps toward translating robotic walking to prostheses: A nonlinear optimization based control approach. Auton. Robot 41,725-742 (2017). 
50. Zhao H, Ambrose E, Ames AD, Preliminary results on energy efficient 3D prosthetic walking with a powered compliant transfemoral prosthesis, in IEEE Int. Conf. Robot. Autom. (ICRA, 2017), pp. 1140-1147.

51. Conghaile PÓ, Falk M, MacAodha D, Yakovleva ME, Gonaus C, Peterbauer CK, Gorton L, Shleev $\mathrm{S}$, Leech D, Fully enzymatic membraneless glucose $\mid$ oxygen fuel cell that provides $0.275 \mathrm{~mA} \mathrm{~cm}^{-2}$ in $5 \mathrm{mM}$ glucose, operates in human physiological solutions, and powers transmission of sensing data. Anal. Chem 88, 2156-2163 (2016). [PubMed: 26750758]

52. Reid RC, Minteer SD, Gale BK, Contact lens biofuel cell tested in a synthetic tear solution. Biosens. Bioelectron 68, 142-148 (2015). [PubMed: 25562741]

53. Falk M, Pankratov D, Lindh L, Arnebrant T, Shleev S, Miniature direct electron transfer based enzymatic fuel cell operating in human sweat and saliva. Fuel Cells 14, 1050-1056 (2014).

54. Falk M, Andoralov V, Blum Z, Sotres J, Suyatin DB, Ruzgas T, Arnebrant T, Shleev S, Biofuel cell as a power source for electronic contact lenses. Biosens. Bioelectron 37, 38-45 (2012). [PubMed: 22621980]

55. Jia W, Wang X, Imani S, Bandodkar AJ, Ramirez J, Mercier PP, Wang J, Wearable textile biofuel cells for powering electronics. J. Mater. Chem. A 2, 18184-18189 (2014). 
A

D
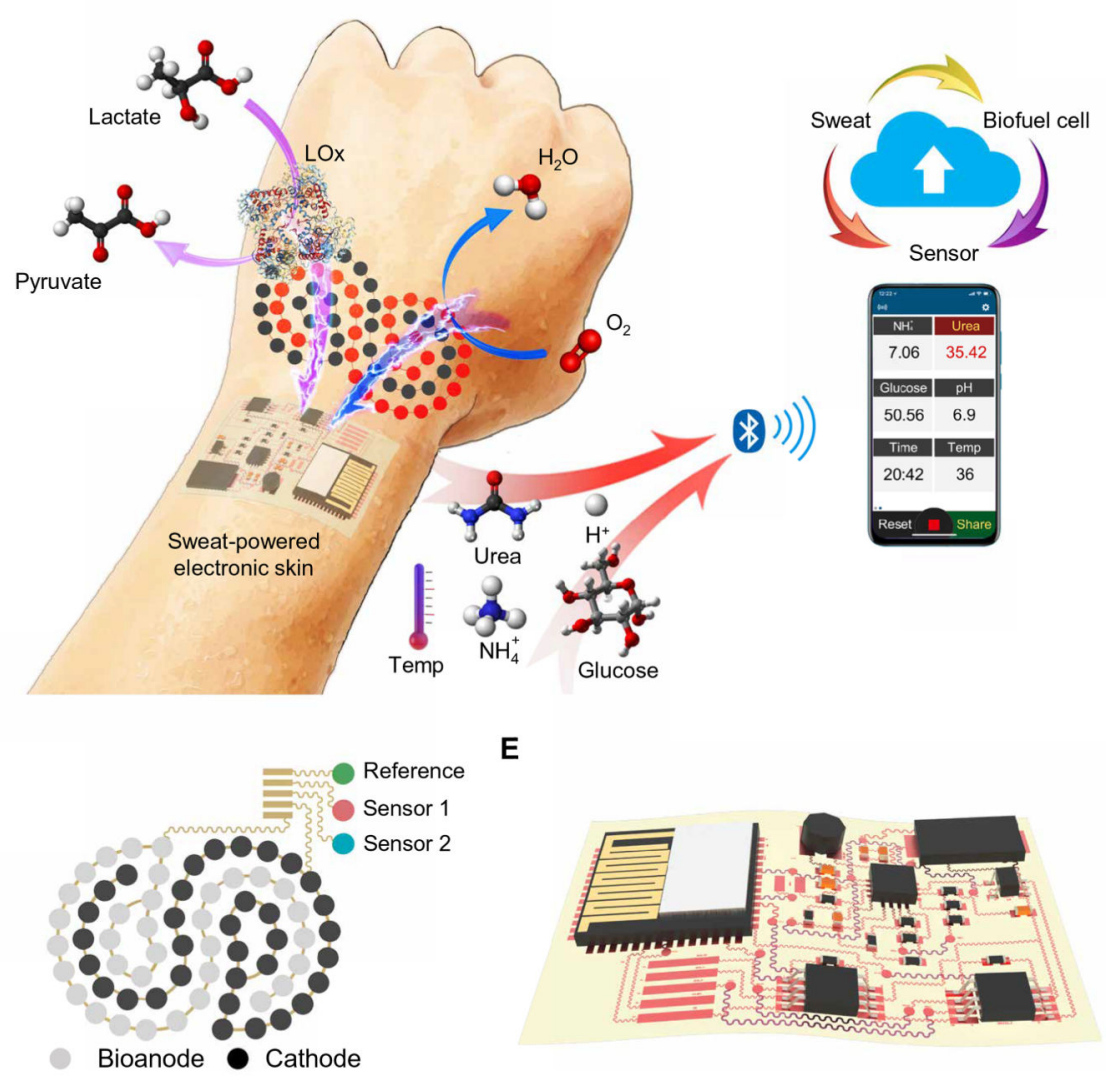

E

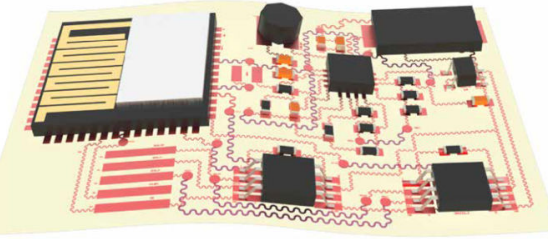
of the PPES for efficient on-body biofluid sampling. M-tape, medical tape.
B

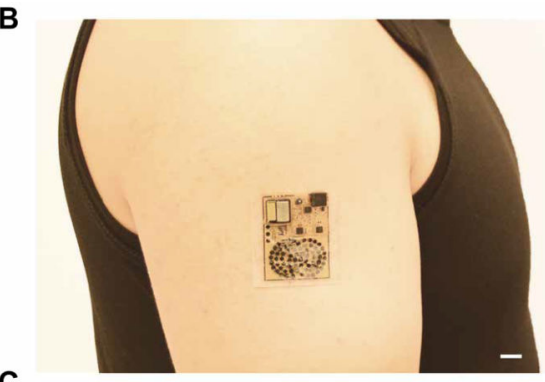

C

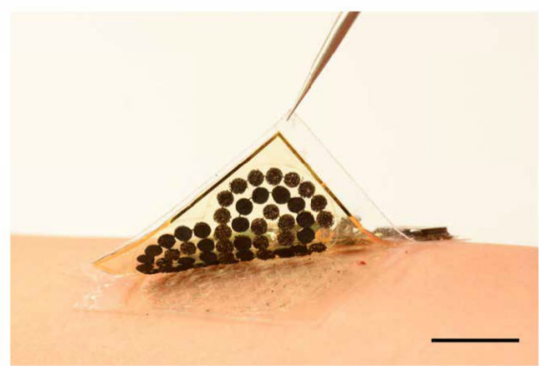

F

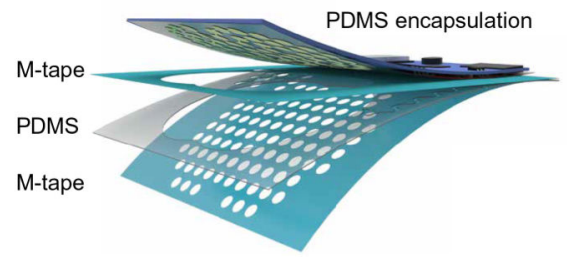

Fig. 1. Perspiration-powered soft electronic skin (e-skin) for multiplexed wireless sensing. (A) Schematic of a battery-free, biofuel-powered e-skin that efficiently harvests energy from the human body, performs multiplexed biosensing, and wirelessly transmits data to a mobile user interface through Bluetooth. (B and C) Photographs of a PPES on a healthy individual's arm. Scale bars, $1 \mathrm{~cm}$. (D and E) Schematic illustrations of the flexible BFC-biosensor patch (D) and the soft electronic-skin interface (E). (F) System-level packaging and encapsulation 


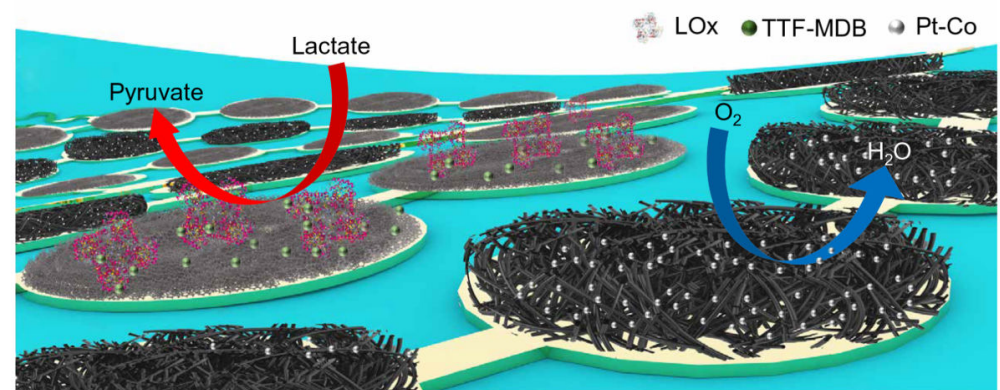

B

A

B

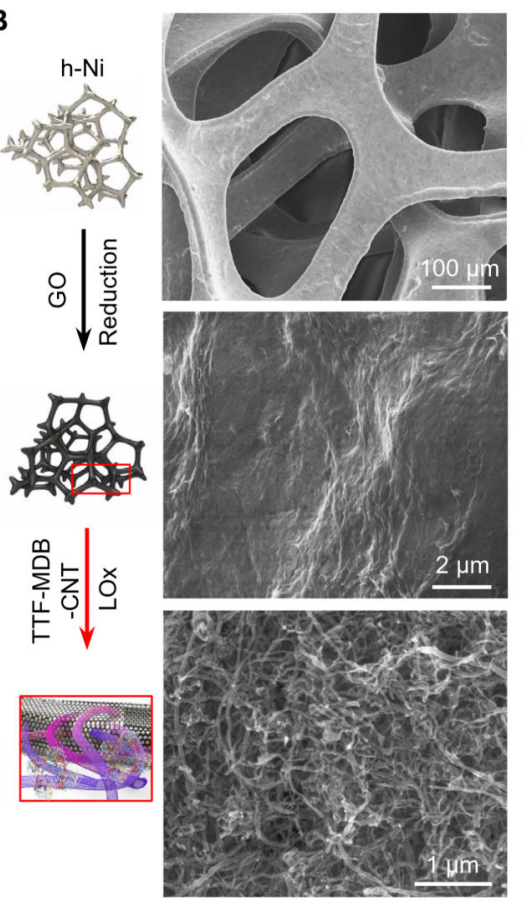

G

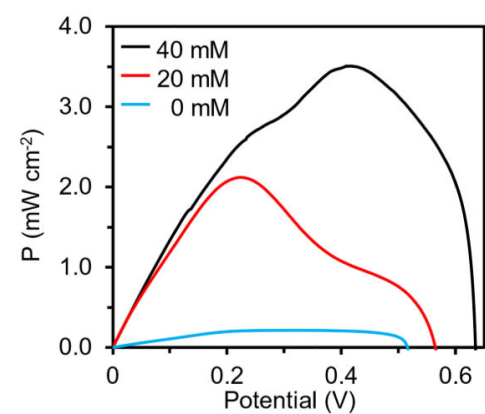

C

MDB-CNT
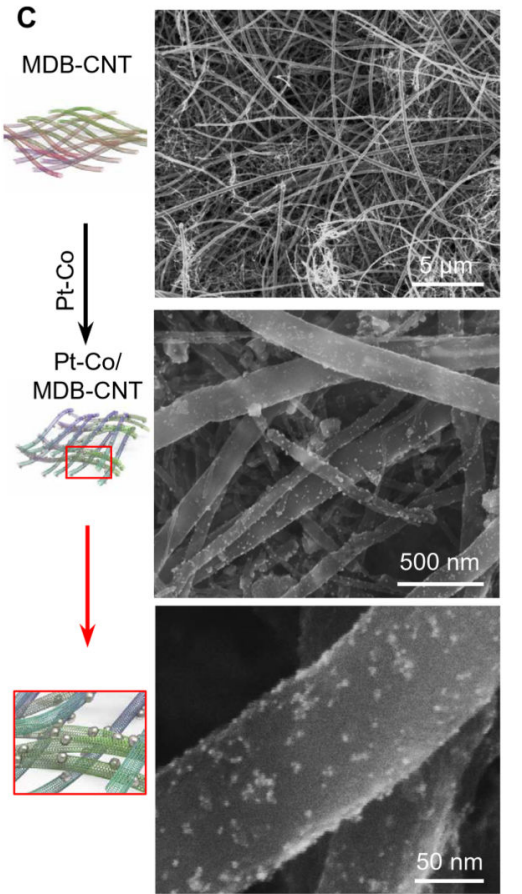

H

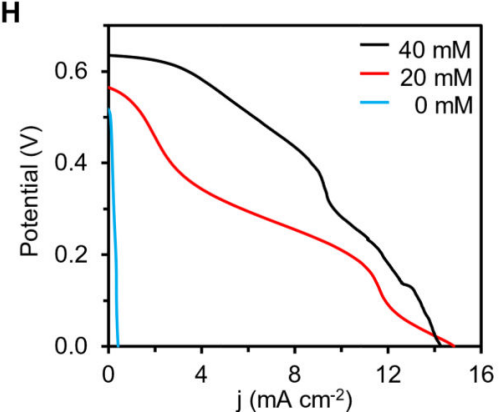

Fig. 2. Flexible nanoengineered BFC array for efficient energy harvesting. nanoparticle-modified cathodes. (B and $\mathbf{C}$ ) Schematic and SEM images showing

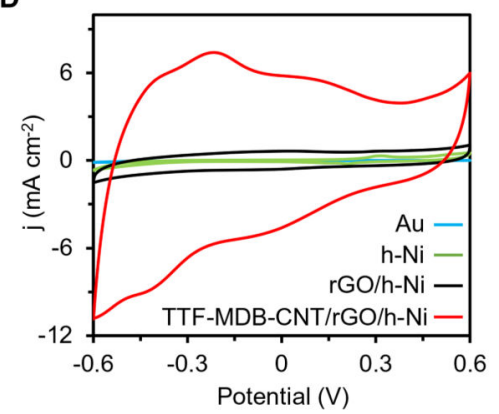

E

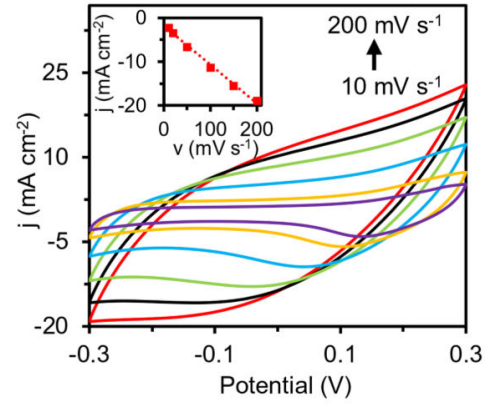

F
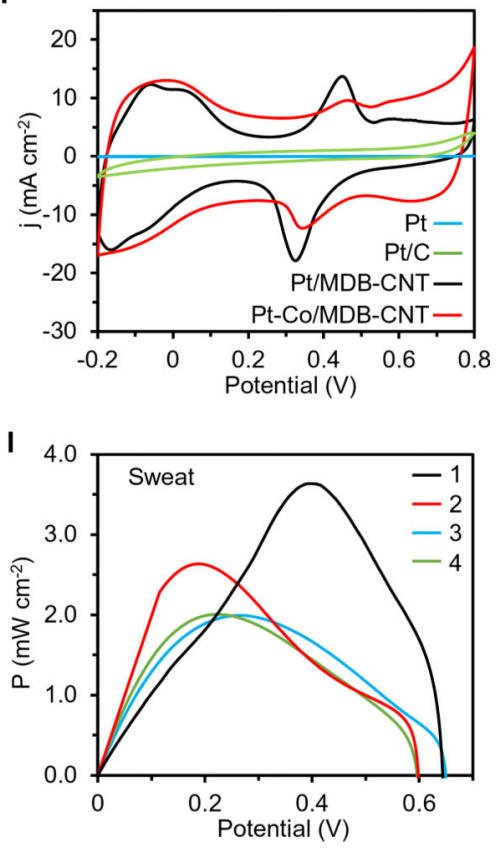

(A) Schematic of a soft BFC array consisting of LOx-modified bioanodes and Pt alloy modification process of bioanode assembled with porous h-Ni, rGO, CNTs, and LOx (B), and cathode assembled with CNT film and Pt alloy nanoparticles (C). (D) CVs of the Au, h$\mathrm{Ni}, \mathrm{rGO} / \mathrm{h}-\mathrm{Ni}$, and MDB-CNT/rGO/h-Ni electrodes. $j$, current density. Scan rate, $50 \mathrm{mV} \mathrm{s}^{-1}$. (E) CVs of the MDB-CNT/rGO/h-Ni with scan rates of 10, 20, 50, 100, 150, and $200 \mathrm{mV} \mathrm{s}$ ${ }^{-1}$. Inset: Calibration curve of the current density at $-0.1 \mathrm{~V}$ versus scan rate (v). (F) CVs of 
the Pt-Co/MDB-CNT, Pt/MDB-CNT, commercial Pt/C, and bulk Pt electrodes recorded in $\mathrm{N}_{2}$-purged $0.1 \mathrm{M} \mathrm{H}_{2} \mathrm{SO}_{4}$ solutions. Pt/C, platinum carbon black. Scan rate, $100 \mathrm{mV} \mathrm{s}^{-1}$. (G and $\mathbf{H})$ Power density $(P)(\mathrm{G})$ and polar curves $(H)$ of BFCs recorded in 0 to $40 \mathrm{mM}$ lactate. Experiments in (D) to $(\mathrm{H})$ were repeated three times independently with similar results. (I) Power density curves of BFCs in sweat samples from four healthy humans. Reference electrodes in (D) to (I), $\mathrm{Ag} / \mathrm{AgCl}$. 
A

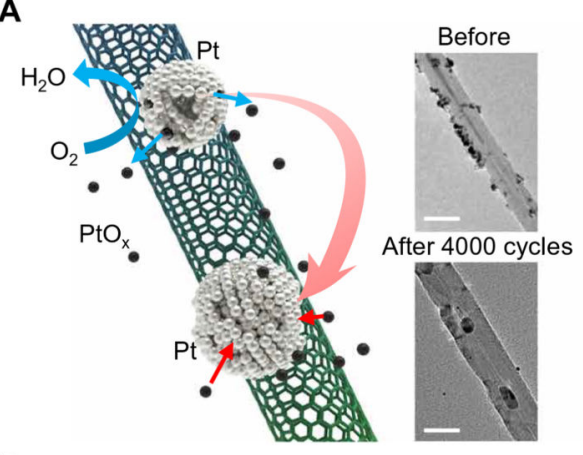

D

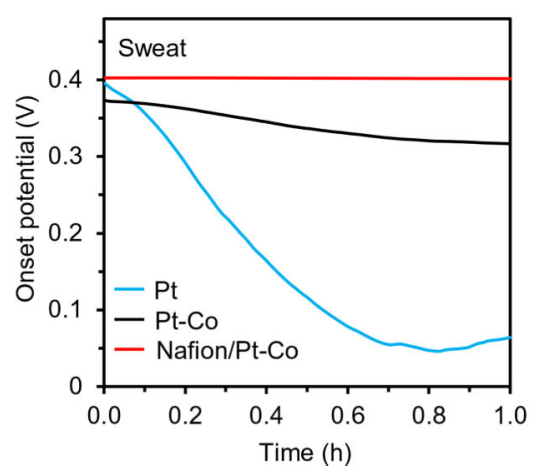

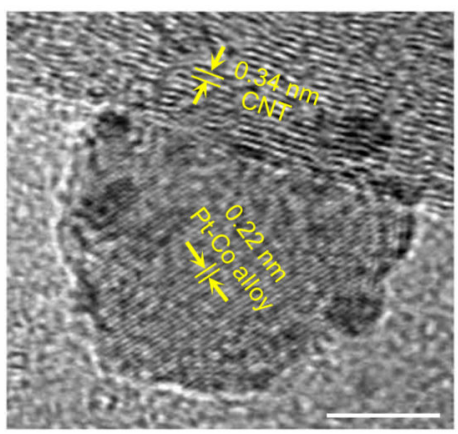

E

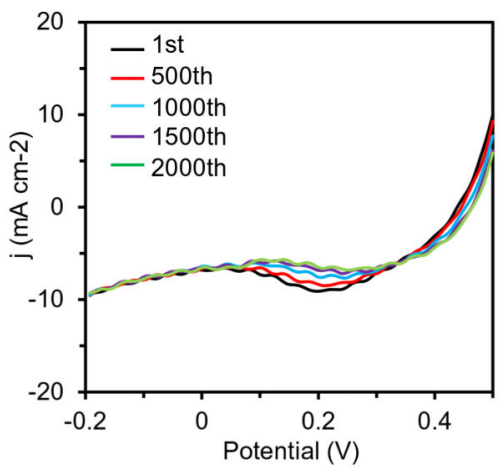

C

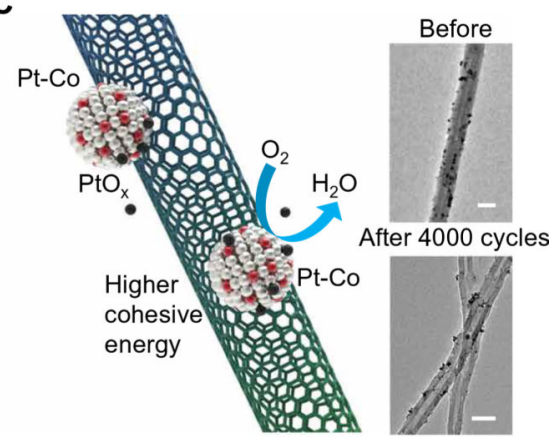

$\mathbf{F}$

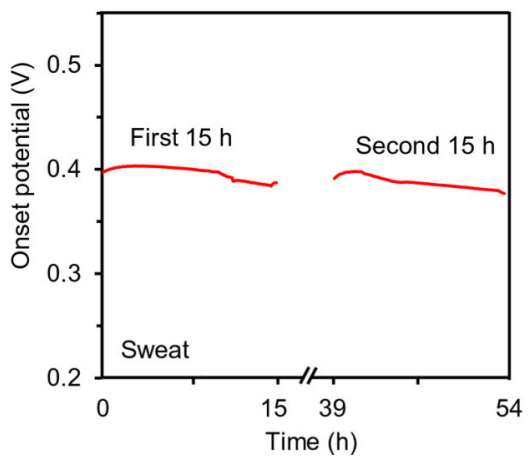

Fig. 3. Characterization of role of Pt alloy nanoparticles in the BFC stability.

(A) Schematic illustration showing that the Pt nanoparticles (decorated on CNTs) merge into larger ones during the prolonged catalysis. Inset: TEM images of the Pt/MDB-CNT before and after $4000 \mathrm{CV}$ cycles between -0.2 and $0.5 \mathrm{~V}$. Scale bars, $50 \mathrm{~nm}$. (B) TEM image showing the crystalline structure of a Pt-Co nanoparticle. Scale bar, $5 \mathrm{~nm}$. (C) Schematic illustration of the Pt-Co alloy nanoparticles on CNT, which maintain the uniform size owing to their higher surface-cohesive energy level. Inset: TEM images of the Pt-Co/MDB-CNT before and after 4000 cycles between -0.2 and $0.5 \mathrm{~V}$. Scale bars, $50 \mathrm{~nm}$. (D) The onset potentials of Pt/MDB-CNT-, Pt-Co/MDB-CNT-, and Nafion/Pt-Co/MDB-CNT-modified cathodes measured in sweat more than a 1-hour period. (E) Repetitive LSVs of a Nafion/PtCo/MDB-CNT-modified cathode obtained during $2000 \mathrm{CV}$ cycles between -0.2 and $0.5 \mathrm{~V}$. (F) Long-term stability test of a Nafion/Pt-Co/ MDB-CNT cathode in sweat more than 30 hours. Reference electrodes in (A) and (C to F), $\mathrm{Ag} / \mathrm{AgCl}$. Experiments in (D) to (F) were repeated three times independently with similar results. 
A

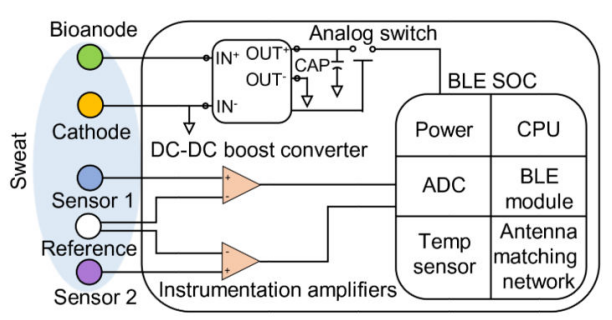

C

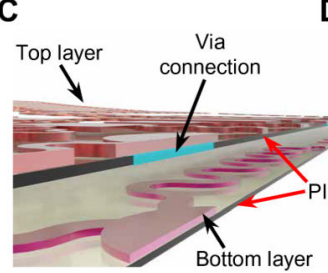

G

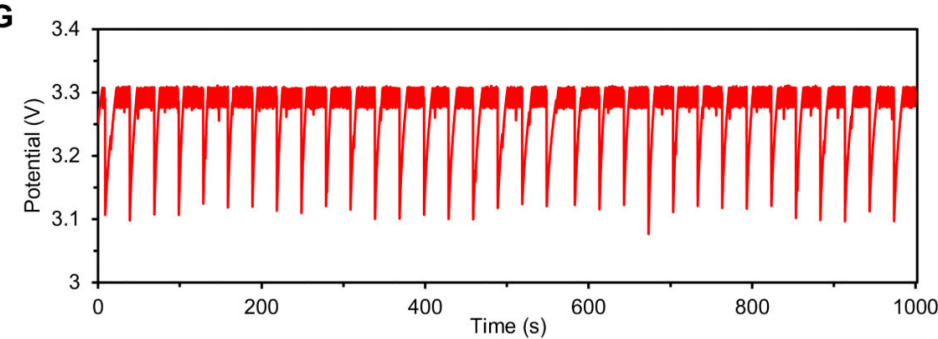

B

E

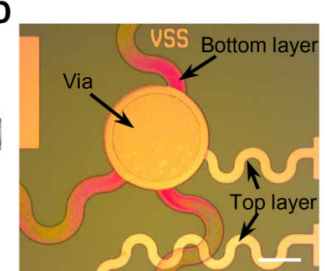

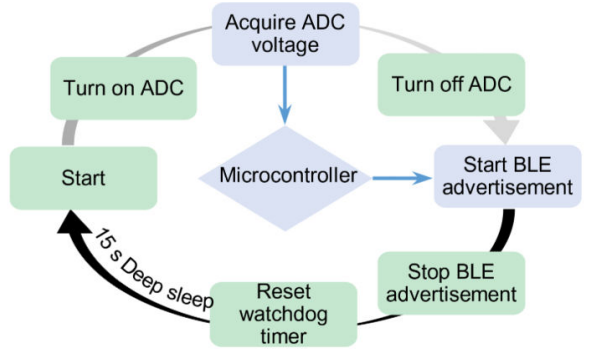

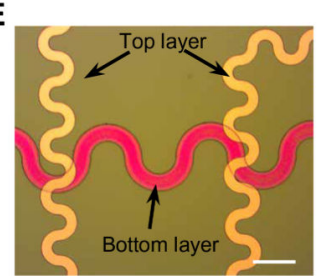

$\mathbf{F}$
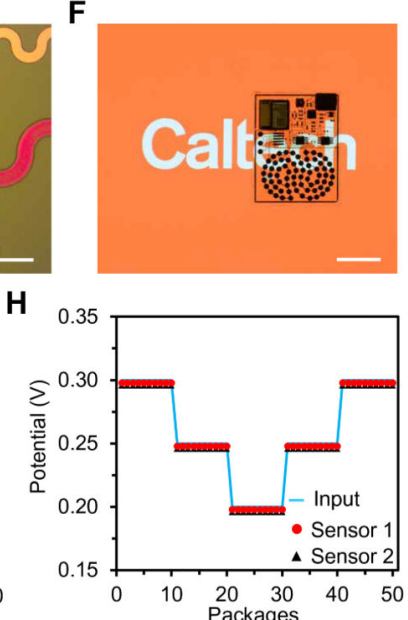

I

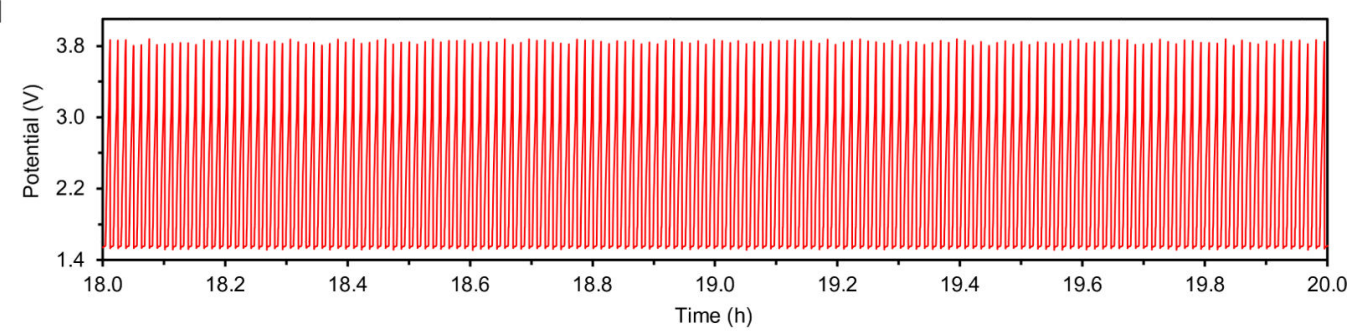

$\mathbf{J}$

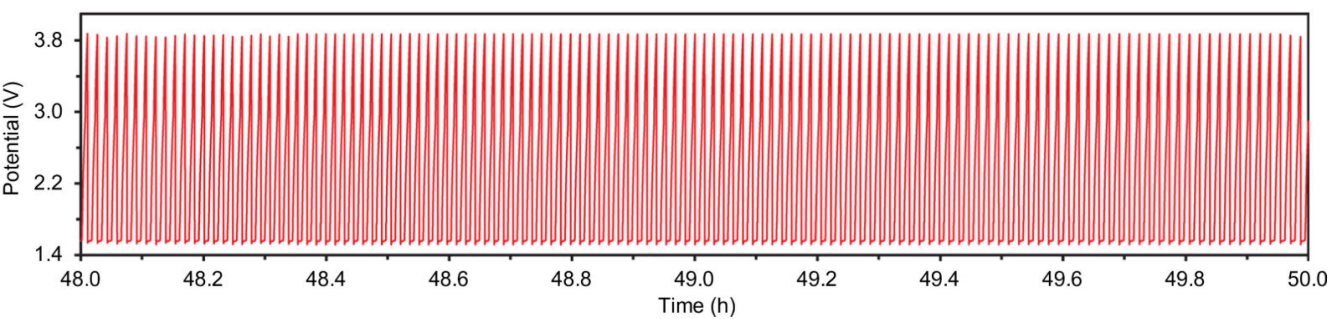

Fig. 4. System-level integration and evaluation of the PPES.

(A) Schematic diagram of the PPES system including the BFC array, sensor array, boost converter, instrumentation amplifiers, and BLE module. SOC, system-on-chip; CPU, central processing unit. (B) Operation flow of the energy control and data-transmission processes. (C to $\mathbf{E}$ ) Schematic (C) and microscopic images (D and E) showing the via structure and soft interconnects of the e-skin system. Scale bars, $3 \mathrm{~mm}$. (F) A photographic image of a fully integrated PPES. Scale bar, $2 \mathrm{~cm}$. (G) Real-time potential of the capacitor measured during continuous operation of a PPES in $20 \mathrm{mM}$ lactate. (H) Characterization of BFC-powered multiplexed sensing with varied electrical inputs in $20 \mathrm{mM}$ lactate. (I and $\mathbf{J}$ ) Long-term stability of the capacitor $(220 \mu \mathrm{F})$-charging process with BFCs through the DC-DC 
converter more than 50 hours in $20 \mathrm{mM}$ lactate. Potential dynamics from 18 to 20 hours and from 48 to 50 hours are illustrated in $(\mathrm{I})$ and $(\mathrm{J})$, respectively. Experiments in $(\mathrm{G})$ to $(\mathrm{J})$ were repeated three times independently with similar results. 
A

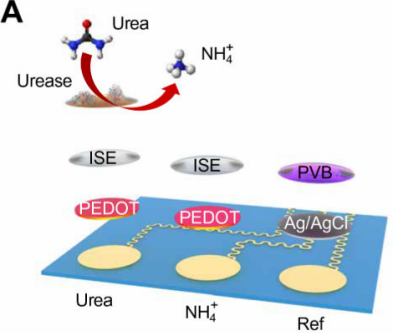

E
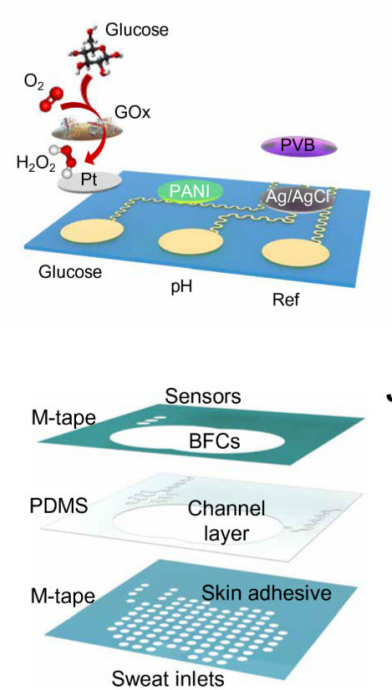

$\mathbf{F}$

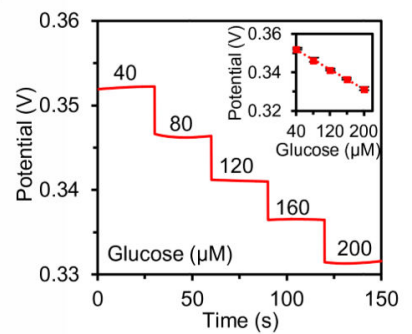

J

B
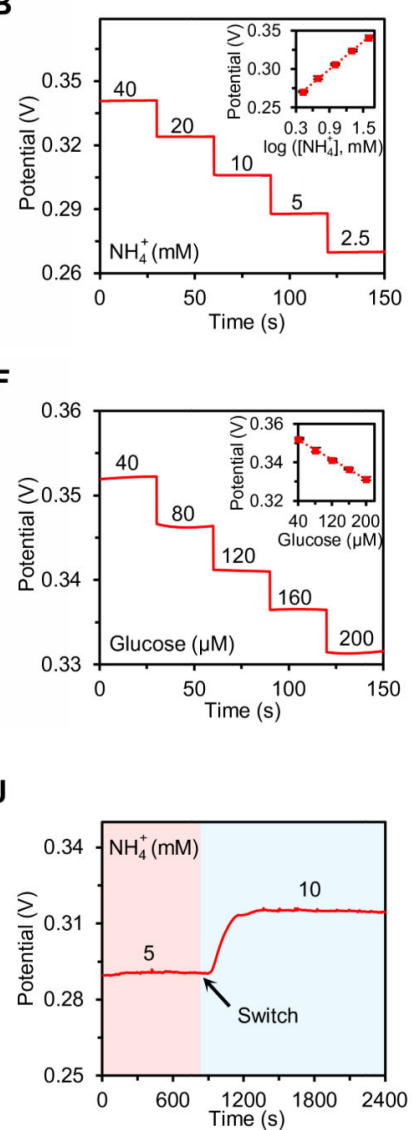

C

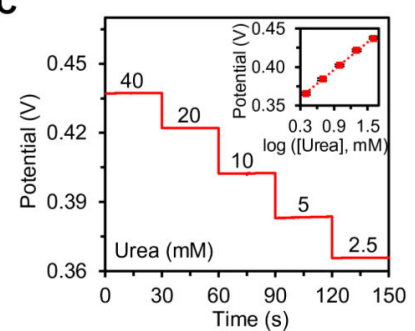

G

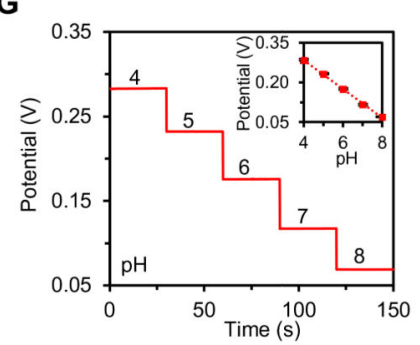

D

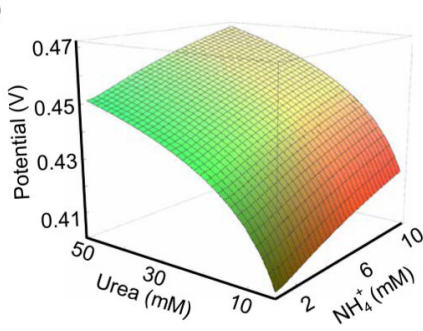

$\mathrm{H}$

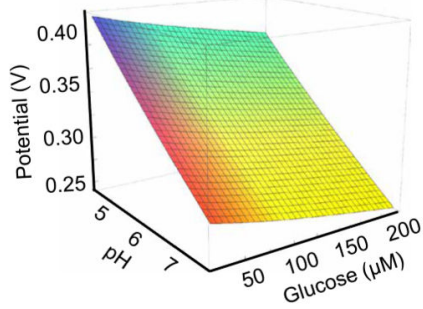

L

K
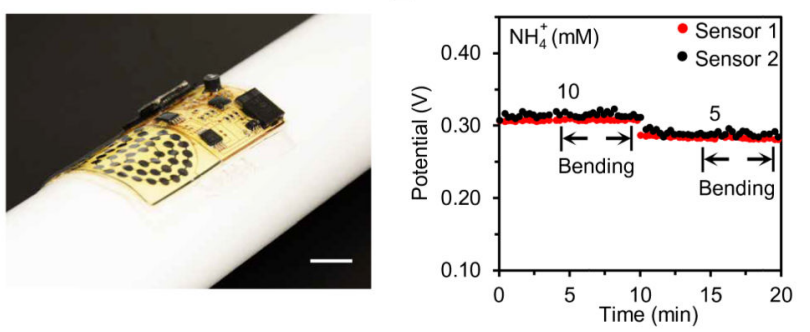
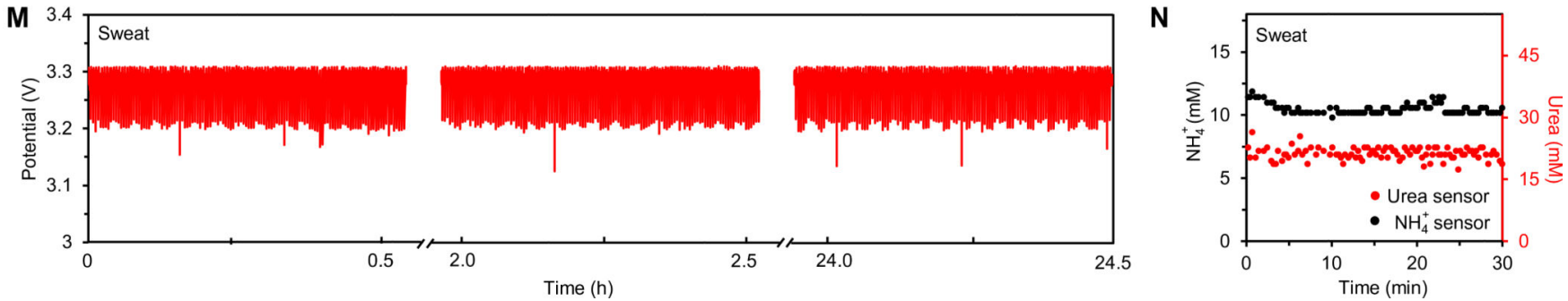

Fig. 5. Characterization of the PPES for multiplexed biosensing.

(A) Schematic of a sensor array for simultaneous urea and $\mathrm{NH}_{4}{ }^{+}$monitoring. (B and $\mathbf{C}$ ) OCP responses of a $\mathrm{NH}_{4}{ }^{+}$sensor (B) and a urea sensor (C) in standard analyte solutions. Insets: The corresponding calibration plots of the $\mathrm{NH}_{4}{ }^{+}$and urea sensors. Data recording was paused for $30 \mathrm{~s}$ for each solution change. Error bars represent the SDs from five sensors. (D) A color map showing the dependence of the urea sensor response on urea and $\mathrm{NH}_{4}{ }^{+}$ concentrations. Red indicates higher $\mathrm{NH}_{4}{ }^{+}$level and green indicates higher urea level. (E) Schematic of a sensor array for simultaneous glucose and $\mathrm{pH}$ sensing. (F and $\mathbf{G}$ ) OCP responses of a glucose sensor $(\mathrm{F})$ and a pH sensor $(\mathrm{G})$ in standard analyte solutions. Insets: The corresponding calibration plots of the glucose and $\mathrm{pH}$ sensors. Data recording was paused for $30 \mathrm{~s}$ for each solution change. Error bars represent the SDs from five sensors. (H) A color map showing the dependence of the glucose sensor on the glucose and $\mathrm{pH}$ levels. Red indicates higher $\mathrm{pH}$ value and green indicates higher glucose level. (I) Schematic of the microfluidic design for biofluid sampling. (J) Dynamic response of an $\mathrm{NH}_{4}{ }^{+}$sensor upon 
switching the inflow solutions at a flow rate of $0.05 \mathrm{ml} \mathrm{hour}^{-1}$. (K) A photograph of an integrated PPES under mechanical deformation. (L) The performance of a two-channel $\mathrm{NH}_{4}{ }^{+}$sensor array under mechanical deformation (with a radius of bending curvature of 1.5 $\mathrm{cm})$. (M and $\mathbf{N}$ ) Potential of the capacitor (M) and responses of a urea and $\mathrm{NH}_{4}{ }^{+}$sensor array $(\mathrm{N})$ when the PPES operates in a human sweat sample. 
A

D

E
B
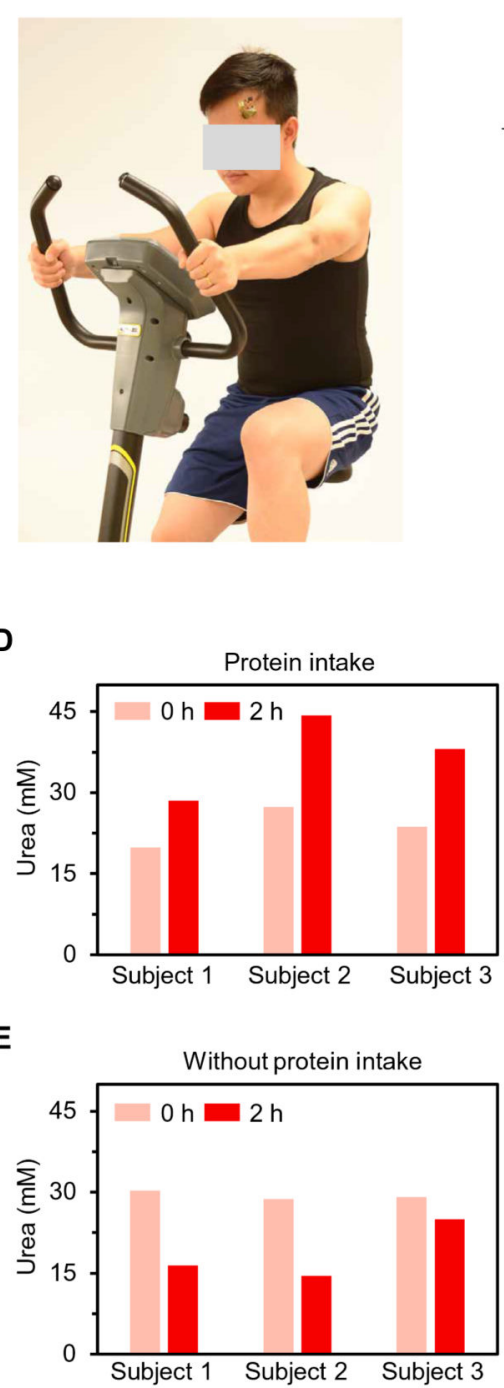

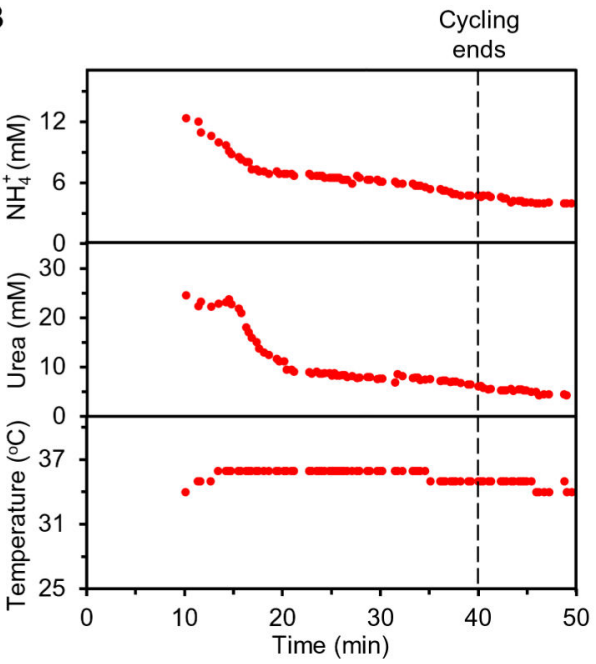

$\mathbf{F}$

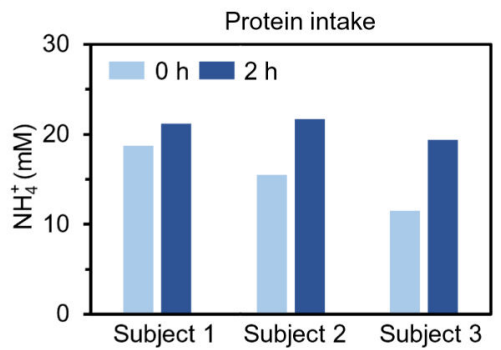

G

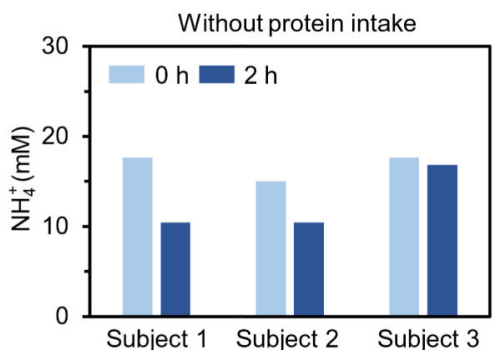

C

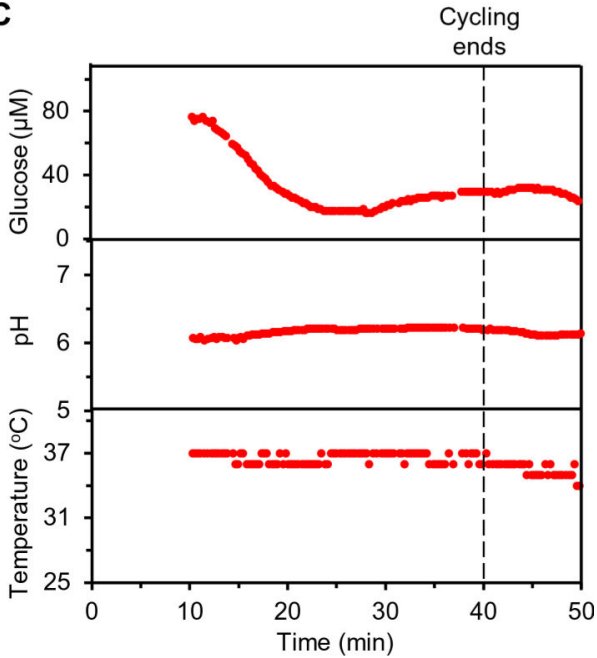

H

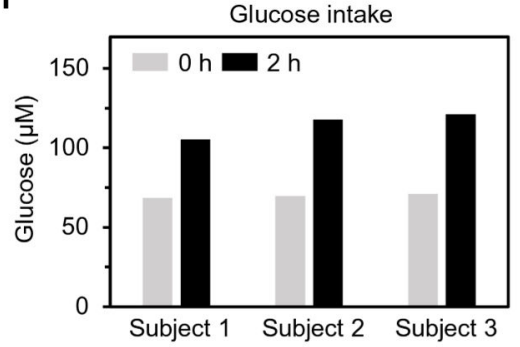

1

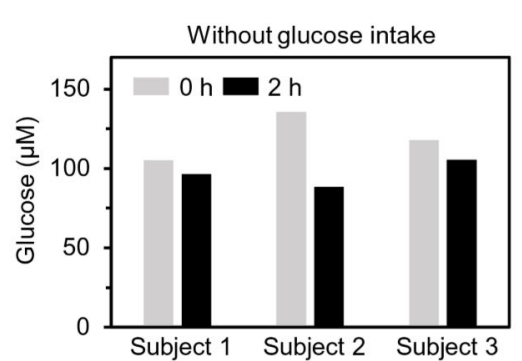

Fig. 6. On-body evaluation of the PPES toward personalized metabolic monitoring.

(A) Photograph of an individual wearing a PPES during cycling exercise. (B) Real-time multiplexed urea and $\mathrm{NH}_{4}{ }^{+}$analysis using a PPES on an individual's forehead. (C) Realtime multiplexed glucose and $\mathrm{pH}$ monitoring using a PPES on an individual's forehead. (D to I) Evaluation of the PPES in dietary challenges: sweat urea levels with (D) and without (E) protein intake, sweat $\mathrm{NH}_{4}{ }^{+}$levels with $(\mathbf{F})$ and without $(\mathbf{G})$ protein intake, and sweat glucose levels with (H) and without (I) glucose intake. 
A
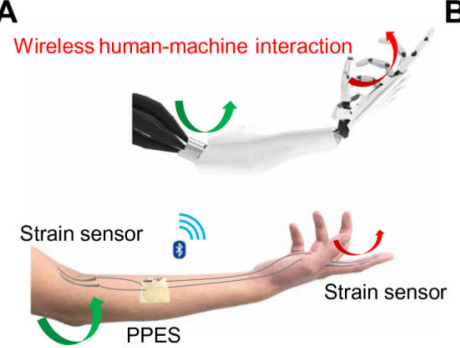

B

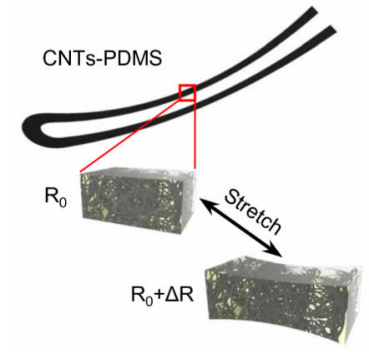

C

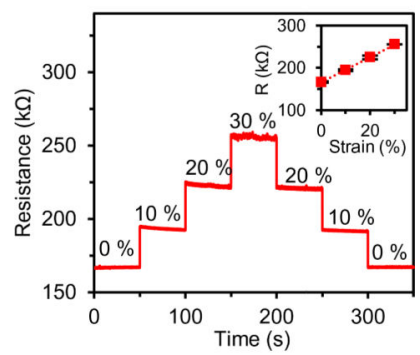

$\mathbf{F}$

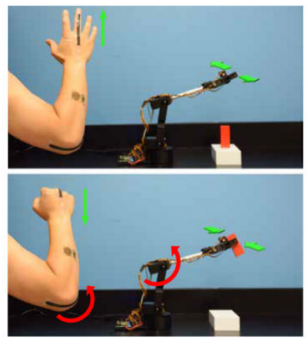

G

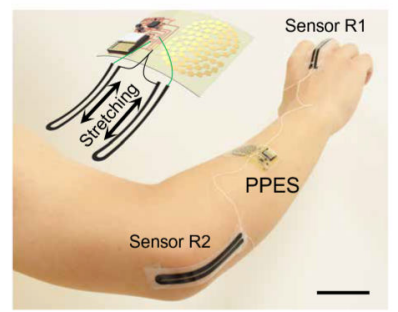

E

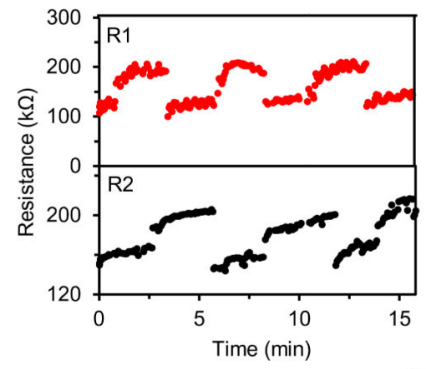

H
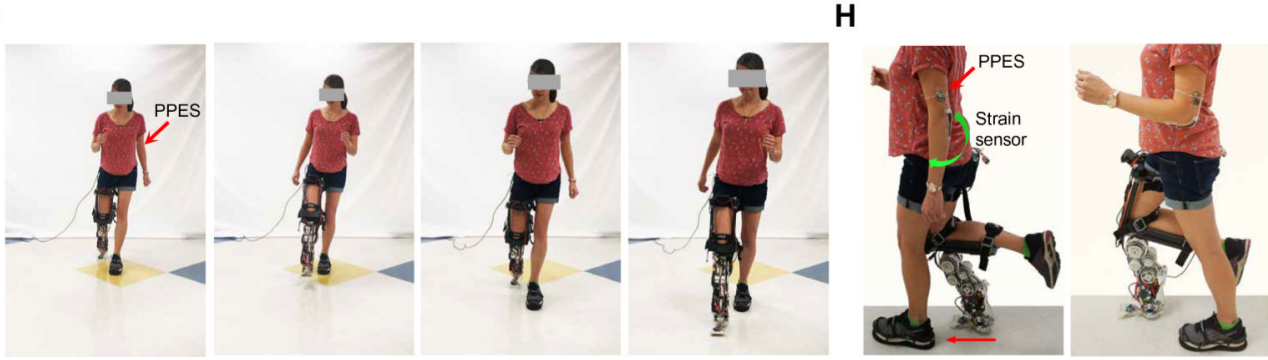

Fig. 7. On-body evaluation of the PPES as a wireless human-machine interface for robotic assistance.

(A) Schematic illustration of the PPES for remote human-machine interaction. (B)

Schematic of the CNTs-PDMS elastomer-based stain sensors. (C) Resistance response of a CNTs-PDMS strain sensor under different strains. (D) Photograph and schematic (inset) of the PPES integrated with strain sensors. (E) Real-time multidegree motion tracking using a PPES with the strain sensors on an individual's finger and elbow. (F) Time-lapse images of the wireless robotic arm control using a PPES. ( $\mathbf{G}$ and $\mathbf{H}$ ) Time-lapse images of front view $(\mathrm{G})$ and side view $(\mathrm{H})$ of the use of the PPES for robotic prosthesis control. 